\title{
Global, Regional, and National Cancer Incidence, Mortality, Years of Life Lost, Years Lived With Disability, and Disability-Adjusted Life-Years for 29 Cancer Groups, 1990 to 2017 A Systematic Analysis for the Global Burden of Disease Study
}

Global Burden of Disease Cancer Collaboration

IMPORTANCE Cancer and other noncommunicable diseases (NCDs) are now widely recognized as a threat to global development. The latest United Nations high-level meeting on NCDs reaffirmed this observation and also highlighted the slow progress in meeting the 2011 Political Declaration on the Prevention and Control of Noncommunicable Diseases and the third Sustainable Development Goal. Lack of situational analyses, priority setting, and budgeting have been identified as major obstacles in achieving these goals. All of these have in common that they require information on the local cancer epidemiology. The Global Burden of Disease (GBD) study is uniquely poised to provide these crucial data.

OBJECTIVE To describe cancer burden for 29 cancer groups in 195 countries from 1990 through 2017 to provide data needed for cancer control planning.

EVIDENCE REVIEW We used the GBD study estimation methods to describe cancer incidence, mortality, years lived with disability, years of life lost, and disability-adjusted life-years (DALYs). Results are presented at the national level as well as by Socio-demographic Index (SDI), a composite indicator of income, educational attainment, and total fertility rate. We also analyzed the influence of the epidemiological vs the demographic transition on cancer incidence.

FINDINGS In 2017, there were 24.5 million incident cancer cases worldwide (16.8 million without nonmelanoma skin cancer [NMSC]) and 9.6 million cancer deaths. The majority of cancer DALYs came from years of life lost (97\%), and only $3 \%$ came from years lived with disability. The odds of developing cancer were the lowest in the low SDI quintile (1 in 7) and the highest in the high SDI quintile (1 in 2) for both sexes. In 2017, the most common incident cancers in men were NMSC (4.3 million incident cases); tracheal, bronchus, and lung (TBL) cancer (1.5 million incident cases); and prostate cancer (1.3 million incident cases). The most common causes of cancer deaths and DALYs for men were TBL cancer (1.3 million deaths and 28.4 million DALYs), liver cancer (572 000 deaths and 15.2 million DALYs), and stomach cancer (542000 deaths and 12.2 million DALYs). For women in 2017, the most common incident cancers were NMSC ( 3.3 million incident cases), breast cancer (1.9 million incident cases), and colorectal cancer (819 000 incident cases). The leading causes of cancer deaths and DALYs for women were breast cancer (601000 deaths and 17.4 million DALYs), TBL cancer ( 596000 deaths and 12.6 million DALYs), and colorectal cancer (414 000 deaths and 8.3 million DALYs).

CONCLUSIONS AND RELEVANCE The national epidemiological profiles of cancer burden in the GBD study show large heterogeneities, which are a reflection of different exposures to risk factors, economic settings, lifestyles, and access to care and screening. The GBD study can be used by policy makers and other stakeholders to develop and improve national and local cancer control in order to achieve the global targets and improve equity in cancer care.
Supplemental content

CME Quiz at

jamacmelookup.com and CME Questions page 1816
Group Information: The members of the Global Burden of Disease Cancer Collaboration appear at the end of the article.

Corresponding Author: Christina Fitzmaurice, MD, MPH, Division of Hematology, Department of Medicine, Institute for Health Metrics and Evaluation, University of Washington, 23015th Ave, Ste 600, Seattle, WA 98121 (cf11@uw.edu). 
C ancer is now widely recognized as a global problem that unfortunately lacks a global solution. The latest United Nations high-level meeting on noncommunicable diseases (NCDs) exemplified this conundrum. ${ }^{1}$ Despite global commitment to reducing the risk of and disability from NCDs, including cancer, implementation of known solutions is inadequate to reach the 2011 Political Declaration on the Prevention and Control of Noncommunicable Diseases ${ }^{2,3}$ (25\% reduction in premature mortality from NCDs by 2025) and the third Sustainable Development Goal (by 2030 reduce by onethird premature mortality from NCDs through prevention and treatment, and promote mental health and well-being). ${ }^{4}$ To reduce cancer burden, identifying the scope of the problem and mapping out implementation of solutions is best done in $\mathrm{Na}-$ tional Cancer Control Plans (NCCPs). However, a recent review showed that only $29 \%$ of low-income countries had a NCCP, and even if NCCPs existed, cost, financing, monitoring, and expansion of information systems was often inadequate. Many highly effective prevention and treatment strategies exist for cancer. However, they are often very specific (eg, vaccination for human papillomavirus and hepatitis B virus for prevention of cervical and liver cancer, or tyrosine kinase inhibitors for cancers with targetable mutations). Effective NCCPs therefore require detailed knowledge about the local burden of cancer and associated risk factors. We herein present results from the Global Burden of Disease (GBD) 2017 study describing cancer incidence, mortality, years of life lost (YLLs), years lived with disability (YLDs), and disability-adjusted lifeyears (DALYs) for 195 countries from 1990 through 2017, which can inform cancer control through policy, resource allocation, and health system planning.

\section{Methods}

Methods have remained similar to the GBD 2016 study. ${ }^{5}$ Detailed descriptions of the methods can be found in the GBD 2017 publications $^{6-9}$ as well as in the eAppendix, eFigures, and eTables in the Supplement. For each GBD study, the entire time series is re-estimated. This study therefore supersedes prior GBD iterations. The GBD study is compliant with the Guidelines for Accurate and Transparent Health Estimates Reporting statement (eTable 1 in the Supplement). Compared with the prior GBD study (GBD 2016), the neoplasms category for GBD 2017 also includes benign and in situ neoplasms (International Statistical Classification of Diseases and Related Health Problems, Tenth Revision [ICD-10] codes D00-D49). Because disability associated with benign neoplasms is most often very small, we only estimated disability for the new cause: myelodysplastic, myeloproliferative, and other hematopoietic neoplasms. The terms malignant neoplasms or cancer in this article only include $I C D-10$ codes COO through C96. Other changes since GBD 2016 are the addition of new data sources (eTable 3 in the Supplement) for GBD 2017 and improvements in the way we estimated cancer survival by using the mortality-to-incidence ratio (MIR). In this study, estimates are presented for 29 cancer categories and 195 countries and territories. Estimates for benign neoplasms as well as selected sub-

\section{Key Points}

Question What is the cancer burden over time at the global and national levels, measured in incidence, mortality, years lived with disability, years of life lost, and disability-adjusted life-years (DALYs), and how does it compare with other diseases?

Findings Results of this systematic analysis show that in 2017 there were 24.5 million incident cases (16.8 million without nonmelanoma skin cancer), 9.6 million deaths, and 233.5 million DALYs due to cancer; between 2007 and 2017, incident cases increased by $33 \%$, with the lowest increase in the most developed countries, and between 1990 and 2017 neoplasms increased among the top causes of DALYs from the sixth to the second place. Fifty-one percent of cancer cases occurred in countries of high Socio-demographic Index, but only $30 \%$ of cancer deaths and $24 \%$ of cancer DALYs.

Meaning To ensure sustainable global development, increased efforts are needed in cancer prevention and in ensuring universal access to cancer care.

national estimates are available online (https://vizhub. healthdata.org/gbd-compare/ and http://ghdx.healthdata.org/ gbd-results-tool). All rates are reported per 100000 personyears. The GBD world population standard was used for the calculation of age-standardized rates. ${ }^{9}$ We report $95 \%$ uncertainty intervals for all estimates.

\section{Estimation Framework}

The GBD cancer estimation process starts with mortality. Mortality estimates are made based on vital registration system ( $83 \%$ of data), cancer registry (16\% of data) (eTable 3 in the Supplement), and verbal autopsy data ( $1 \%$ of data) using an ensemble model approach. ${ }^{9,10}$ Predictive covariates used in the model can be found in the eAppendix (eTable 8 in the Supplement). Single-cause mortality estimates are scaled into the separately estimated all-cause estimate. ${ }^{9}$ To estimate cancer incidence, mortality estimates are divided by a separately estimated MIR for each cancer type, sex, 5-year age group, location, and year; additional information regarding incidence and MIR estimation can be found in the eAppendix and eFigure 2 in the Supplement. Data sources used for estimating MIRs are described in eTable 2 in the Supplement. MIRs allow for a uniform method to estimate incidence. Other cancer estimation frameworks ${ }^{11,12}$ have set a precedent for using MIRs for decades and have detailed its benefits, including greater representativeness, especially in settings that lack quality or complete population-based cancer registry systems. By determining incidence using mortality, we are able to account for uncaptured incident cases and, if mortality and incidence are determined correctly, estimating incidence based on MIRs should result in the similar results if using incidence directly. The correlation between survival data and the MIR is used to estimate 10-year cancer prevalence. Total prevalence is partitioned into 4 sequelae: (1) diagnosis/treatment, (2) remission, (3) metastatic/disseminated, and (4) terminal phase. Each sequela prevalence is multiplied by a disability weight to estimate YLDs. Lifetime prevalence of procedure-related disability is estimated for larynx, breast, colorectal, bladder, and 
prostate cancers. A standard life expectancy is used to estimate years of life lost (YLLs). ${ }^{9}$ DALYs are the sum of YLDs and YLLs. To determine the contribution of population aging, population growth, and change in age-specific rates on the change in incident cases between 2007 and 2017, we use hypothetical demographic scenarios holding 2 of these 3 components constant. Results are stratified by quintiles of Sociodemographic Index (SDI), which is a composite indicator including fertility, education, and income. ${ }^{7}$

\section{Results}

Global Incidence, Mortality, and DALYs In 2017, there were 24.5 million (95\% UI, 22.0-27.4 million) incident cancer cases worldwide and 9.6 million (95\% UI, 9.49.7 million) cancer deaths (Table). Cancer caused 233.5 million (95\% UI, 228.8-238.0 million) DALYs in 2017, of which 97\% came from YLLs and 3\% came from YLDs (eTable 15 and eFigure 4 in the Supplement). Globally, the odds of developing cancer during a lifetime (ages 0-79 years) were 1 in 3 for men and 1 in 4 for women (eTable 16 in the Supplement). These odds differ substantially among SDI quintiles, ranging from 1 in 7 at the lowest SDI quintile to 1 in 2 at the highest SDI quintile for both sexes. In 2017, skin; tracheal, bronchus, and lung (TBL); and prostate cancers were the most common incident cancers in men, accounting for $54 \%$ of all cancer cases. The most common causes of cancer deaths and DALYs for men were TBL, liver, and stomach cancers (Table). For women in 2017, the most common incident cancers were nonmelanoma skin cancer (NMSC), breast cancer, and colorectal cancer, accounting for $54 \%$ of all incident cases. The leading causes of cancer deaths and DALYs for women were breast, TBL, and colorectal cancers.

Between 2007 and 2017, the average annual agestandardized incidence rates (ASIRs) for all cancers combined increased in 123 of 195 countries (Figure 1 and eFigure 5 in the Supplement). In contrast, the average annual agestandardized death rates for all cancers combined decreased within that timeframe in 145 of 195 countries (Figure 2 and eFigure 6 in the Supplement). Incident cases for both sexes combined increased in all SDI quintiles between 2007 and 2017 for nearly all cancers (eTable 14 in the Supplement). The largest increase in cancer incident cases between 2007 and 2017 occurred in middle SDI countries, with a $52 \%$ increase, of which changing age structure contributed $24 \%$, population growth $10 \%$, and changing age-specific incidence rates $18 \%$. The drivers behind increasing cancer incidence differ substantially by SDI. Whereas in the lowest SDI quintile, population growth is the major contributor to the increase in total cancer incidence, in low-middle SDI countries aging and changes in incidence rates contribute equally (each $12 \%$ ), and in highmiddle and high SDI countries, increased incidence is mainly driven by population aging (eTable 14 in the Supplement).

\section{Global Top 10 Cancers in 2017}

The global top 10 cancers were ranked by the highest number of incident cases, excluding "other malignant neoplasms."

\section{Nonmelanoma Skin Cancer}

In 2017, there were 7.7 million (95\% UI, 5.3-10.6 million) incident cases of NMSC, of which 5.9 million (95\% UI, 3.7-8.7 million) were due to basal cell carcinoma and 1.8 million (95\% UI, 1.1-2.6 million) due to squamous cell carcinoma. There were 65000 (95\% UI, 63 000-66 000) deaths due to NMSC (Table) and 1.3 million (95\% UI, 1.3-1.4 million) DALYs, of which $97 \%$ came from YLLs (Figure 3) and 3\% from YLDs (eTable 15 and eFigure 4 in the Supplement). Over a lifetime, the odds of developing NMSC were 1 in 7 for men and 1 in 10 for women globally. For men, the odds ranged from 1 in 71 in low SDI countries to 1 in 2 in high SDI countries, and for women from 1 in 104 in low SDI countries to 1 in 4 in high SDI countries (eTable 16 in the Supplement). An aging and growing population has led to a $33 \%$ (95\% UI, 29\%-36\%) increase in NMSC cancer cases, from 5.8 million (95\% UI, 4.1-7.8 million) in 2007 to 7.7 million (95\% UI, 5.3-10.6 million) in 2017. The majority of this increase $(20 \%)$ can be attributed to a change in the population age structure, and $13 \%$ can be attributed to population growth (eTable 14 and eFigure 11 in the Supplement).

\section{Tracheal, Bronchus, and Lung Cancer}

In 2017, there were 2.2 million (95\% UI, 2.1-2.2 million) incident cases of TBL cancer and 1.9 million (95\% UI, 1.8-1.9 million) deaths. Tracheal, bronchus, and lung cancer caused 40.9 million (95\% UI, 40.0-41.9 million) DALYs in 2017, of which 99\% came from YLLs and 1\% from YLDs (eTable 15 and eFigure 4 in the Supplement). Men were more likely to develop TBL cancer over a lifetime than women ( 1 in 17 men vs 1 in 43 women) (eTable 16 in the Supplement). The odds were the highest in high-middle SDI countries for men (1 in 13) and in high SDI countries for women ( 1 in 28). In low SDI countries, the odds were the lowest ( 1 in 45 for men and 1 in 142 for women). Tracheal, bronchus, and lung cancer was the leading cause of cancer in high-middle SDI countries (eFigure 5 in the Supplement). It was the most common cause of cancer deaths by absolute cases globally, as well as in all SDI quintiles (eFigure 6 in the Supplement). For men, TBL cancer was the most common incident cancer in 48 countries and the most common cause for cancer deaths in 110 countries (eFigures 7 and 9 in the Supplement). For women, TBL cancer was the most common incident cancer in Greenland and the most common cause of cancer deaths in 22 countries (eFigures 8 and 10 in the Supplement). Between 2007 and 2017, TBL cancer cases increased by $37 \%$ (95\% UI, 33\%-40\%). Changing age structure contributed $19 \%$, population growth $13 \%$, and changes in age-specific incidence rates 5\% (eTable 14 and eFigure 11 in the Supplement). The ASIRs between 1990 and 2017 show diverging results between men and women globally and in high SDI countries, with ASIRs decreasing in men but increasing in women (eFigure 12 in the Supplement). In high-middle SDI countries, ASIRs remained stable for men but increased for women, whereas rates increased for both sexes in middle SDI countries (eFigures 13 and 14 in the Supplement).

\section{Breast Cancer}

Breast cancer was the third most common incident cancer overall with an estimated 2.0 million (95\% UI, 1.9-2.0 million) in- 


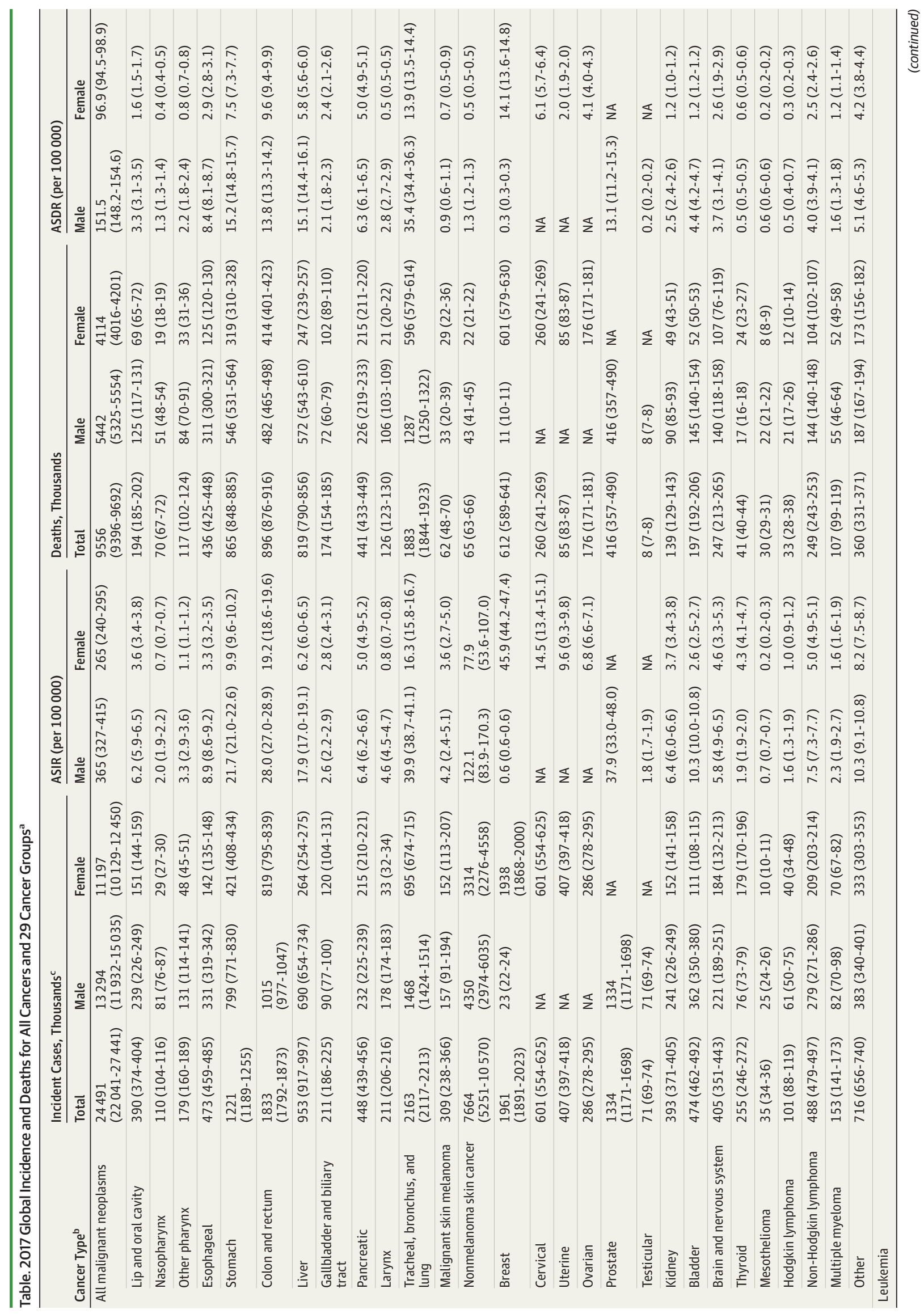




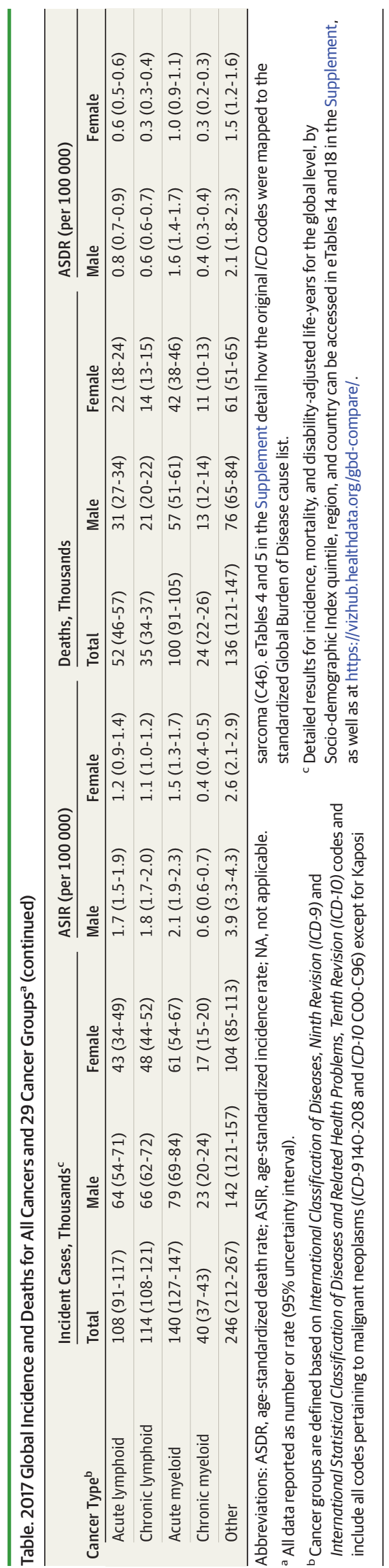

jamaoncology.com 


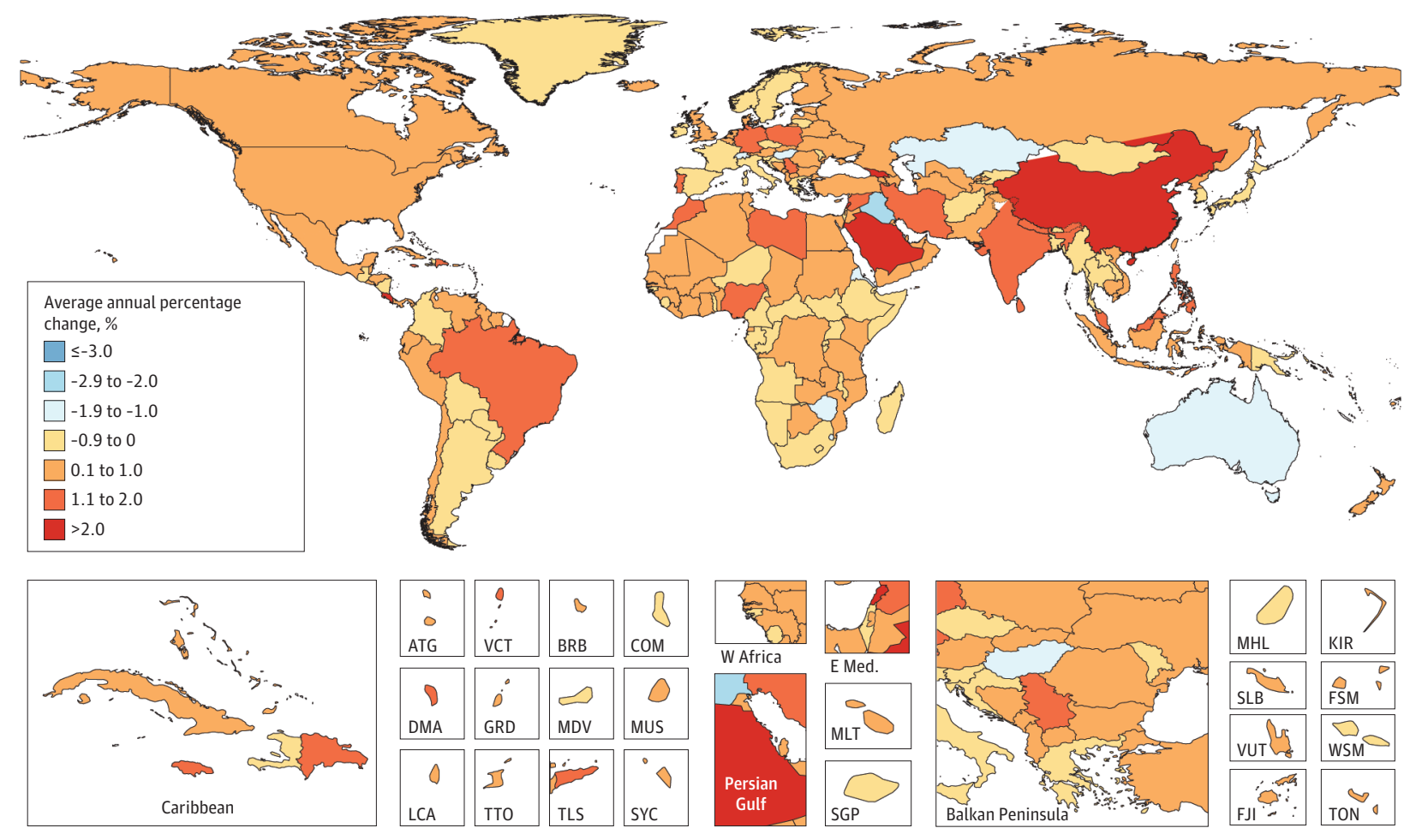

ATG indicates Antigua and Barbuda; BRB, Barbados; COM, Comoros; DMA Dominica; E Med., Eastern Mediterranean; FJI, Fiji; FSM, Federated States of Micronesia; GRD, Grenada; KIR, Kiribati; LCA, Saint Lucia; MDV, Maldives; MLT Malta; MUS, Mauritius; MHL, Marshall Islands; SGP, Singapore; SLB, Solomon
Islands; SYC, Seychelles; TLS, Timor-Leste; TON, Tonga; TTO, Trinidad and Tobago; VCT, Saint Vincent and the Grenadines; VUT, Vanuatu; W Africa, West Africa; WSM, Samoa. cident cases in 2017. The majority occurred in women (1.9 million [95\% UI, 1.9 -2.0 million]) (Table). Breast cancer was among the top 3 leading causes of cancer in all SDI quintiles except for the high and high-middle SDI quintiles, where it was the fourth most common cancer (eFigure 5 in the Supplement). It caused 601000 (95\% UI, $579000-630000$ ) deaths in women and 11000 (95\% UI, 10 000-11 000) deaths in men, making it the fifth leading cause of cancer deaths for both sexes combined in 2017 globally (eFigure 6 in the Supplement). For women, breast cancer was the leading cause of cancer death in 2017 (Table). Breast cancer caused 17.7 million (95\% UI, 16.918.7 million) DALYs for both sexes, of which 93\% came from YLLs and 7\% from YLDs (eTable 15 and eFigure 4 in the Supplement). Globally, 1 in 18 women developed breast cancer over a lifetime (eTable 16 in the Supplement). For women, the odds of developing breast cancer were the highest in high SDI countries (1 in 11), and the lowest in low SDI countries (1 in 38). For women, breast cancer was the most common cancer in 143 countries and the most common cause of cancer deaths in 112 countries (eFigures 8 and 10 in the Supplement). Overall, incident cases increased by 35\% (95\% UI, 30\%-39\%) because of a change in the population age structure (contributing 15\%), population growth (contributing 13\%), and an increase in agespecific incidence rates (contributing 7\%) (eFigure 11 in the Supplement). Between 2007 and 2017, ASIRs for women de- creased in high SDI countries but increased in the other SDI quintiles (eFigures 12-16 in the Supplement).

\section{Colon and Rectum Cancer}

In 2017, there were 1.8 million (95\% UI, 1.8-1.9 million) incident cases of colon and rectum cancer, and 896000 (95\% UI, $876000-916000)$ deaths (Table). Colon and rectum cancer caused 19.0 million (95\% UI, 18.5-19.5 million) DALYs in 2017, of which 95\% came from YLLs and 5\% from YLDs (eTable 15 and eFigure 4 in the Supplement). The odds of developing colon and rectum cancer globally were higher for men than for women ( 1 in 26 for men vs 1 in 40 for women) (eTable 16 in the Supplement). The highest odds were in the high SDI quintile ( 1 in 15 for men and 1 in 25 for women) and the lowest in the low SDI quintile (1 in 81 for men and 1 in 98 for women). Between 2007 and 2017, incidence increased by 38\% (95\% UI, $34 \%-41 \%$ ), from 1.3 million (95\% UI, 1.3-1.3 million) to 1.8 million (95\% UI, 1.8-1.9 million) cases (eTable 14 in the Supplement). Most of this increase can be explained by an aging and growing population ( $20 \%$ and $13 \%$, respectively); however, even with the same population size and age structure, colorectal cancer cases would have increased by 5\% between 2007 and 2017 owing to changing age-specific incidence rates. The ASIRs between 1990 and 2017 are similar for men and women 


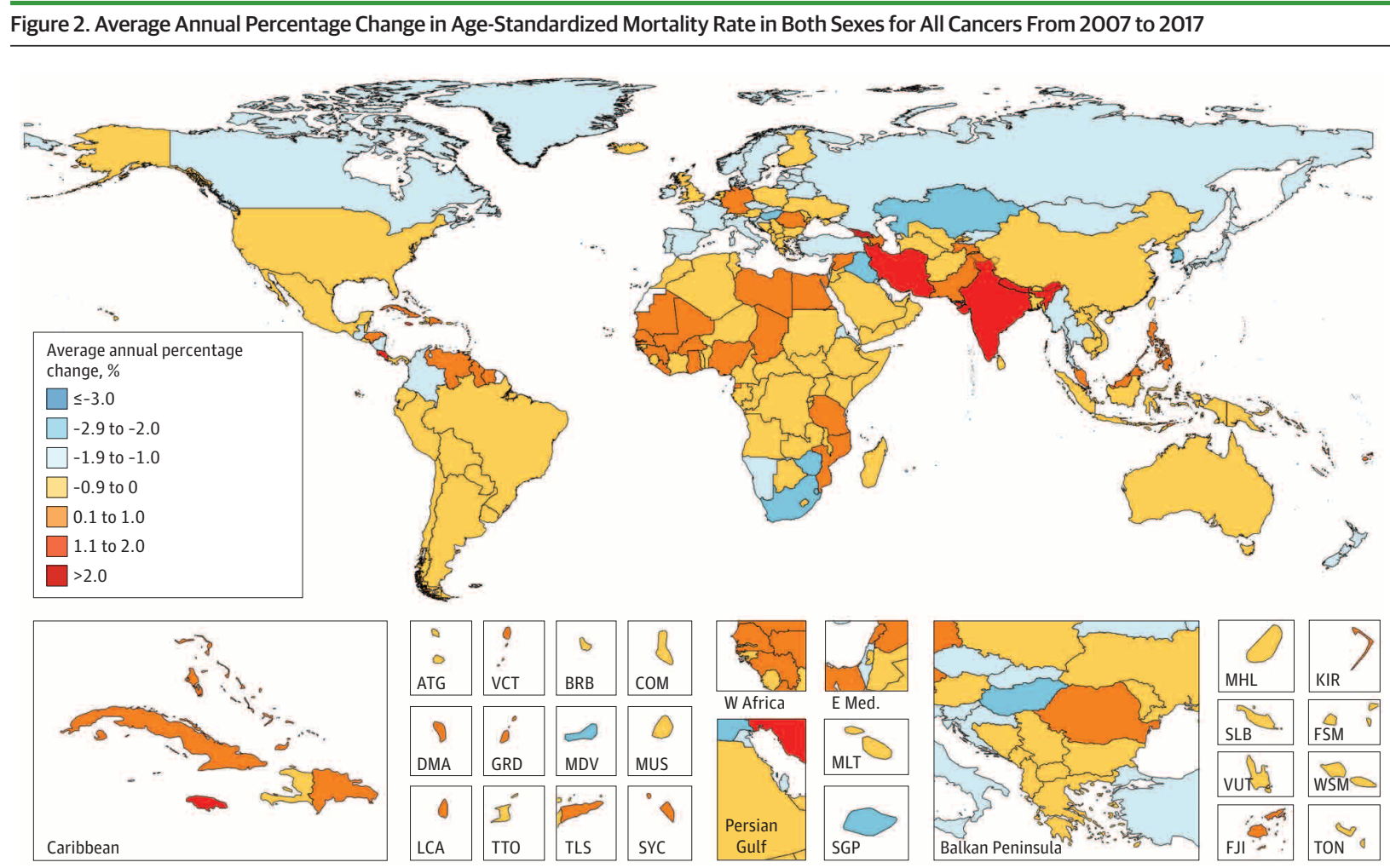

ATG indicates Antigua and Barbuda; BRB, Barbados; COM, Comoros; DMA, Dominica; E Med., Eastern Mediterranean; FJI, Fiji; FSM, Federated States of Micronesia; GRD, Grenada; KIR, Kiribati; LCA, Saint Lucia; MDV, Maldives; MLT, Malta; MUS, Mauritius; MHL, Marshall Islands; SGP, Singapore; SLB, Solomon
Islands; SYC, Seychelles; TLS, Timor-Leste; TON, Tonga; TTO, Trinidad and Tobago; VCT, Saint Vincent and the Grenadines; VUT, Vanuatu; W Africa, West Africa; WSM, Samoa. at the global level and for all SDI quintiles (eFigures 12-16 in the Supplement).

\section{Prostate Cancer}

In 2017, there were 1.3 million (95\% UI, 1.2-1.7 million) incident cases of prostate cancer and 416000 (95\% UI, 357 000490 000) deaths. Prostate cancer caused 7.1 million (95\% UI, 6.1 million-8.4 million) DALYs globally in 2017, with 88\% coming from YLLs and 12\% from YLDs (eTable 15 and eFigure 4 in the Supplement). Globally, the odds of developing prostate cancer were 1 in 18, ranging from 1 in 52 for low SDI countries to 1 in 9 in high SDI countries (eTable 16 in the Supplement). In 2017, prostate cancer was the cancer with the highest incidence for men in 114 countries and the leading cause of cancer-related deaths for men in 56 countries (eFigures 7 and 9 in the Supplement). The increasing incidence rates, together with an aging and growing population, have led to a $42 \%$ (95\% UI, 37\%$52 \%)$ increase in prostate cancer cases since 2007 (940 000 [95\% UI, 774 000-1.2 million] in 2007 and 1.3 million [95\% UI, 1.2-1.7 million] in 2017). Twenty-one percent of this increase can be attributed to a change in the population age structure, $13 \%$ to a change in the population size, and $8 \%$ to a change in the age-specific incidence rates (eTable 14 and eFigure 11 in the Supplement).

\section{Stomach Cancer}

In 2017, there were 1.2 million (95\% UI, 1.2-1.3 million) incident cases of stomach cancer and 865000 (95\% UI, 848000 $885000)$ deaths worldwide. Stomach cancer caused 19.1 million (95\% UI, 18.7-19.6 million) DALYs in 2017, with 98\% coming from YLLs and $2 \%$ coming from YLDs (eTable 15 and eFigure 4 in the Supplement). One in 33 men and 1 in 78 women developed stomach cancer over a lifetime. The highest odds for men and women were in high-middle SDI countries ( 1 in 21 and 1 in 57, respectively), and the lowest odds were for men in low SDI countries ( 1 in 78) and for women in low-middle SDI countries (1 in 104) (eTable 16 in the Supplement). Between 2007 and 2017, stomach cancer moved from the second leading cause of crude cancer YLLs to the third place with a 5\% (95\% UI, 2\%-7\%) increase in absolute YLLs (Figure 3). Overall, incidence between 2007 and 2017 increased by 25\% (95\% UI, 22\%$29 \%$ ), of which a change in the population age structure contributed $19 \%$, population growth $13 \%$, and falling agespecific rates $-6 \%$ (eTable 14 and eFigure 11 in the Supplement). The ASIRs have dropped substantially since 1990 globally and for all SDI quintiles (eFigures 12-16 in the Supplement). 


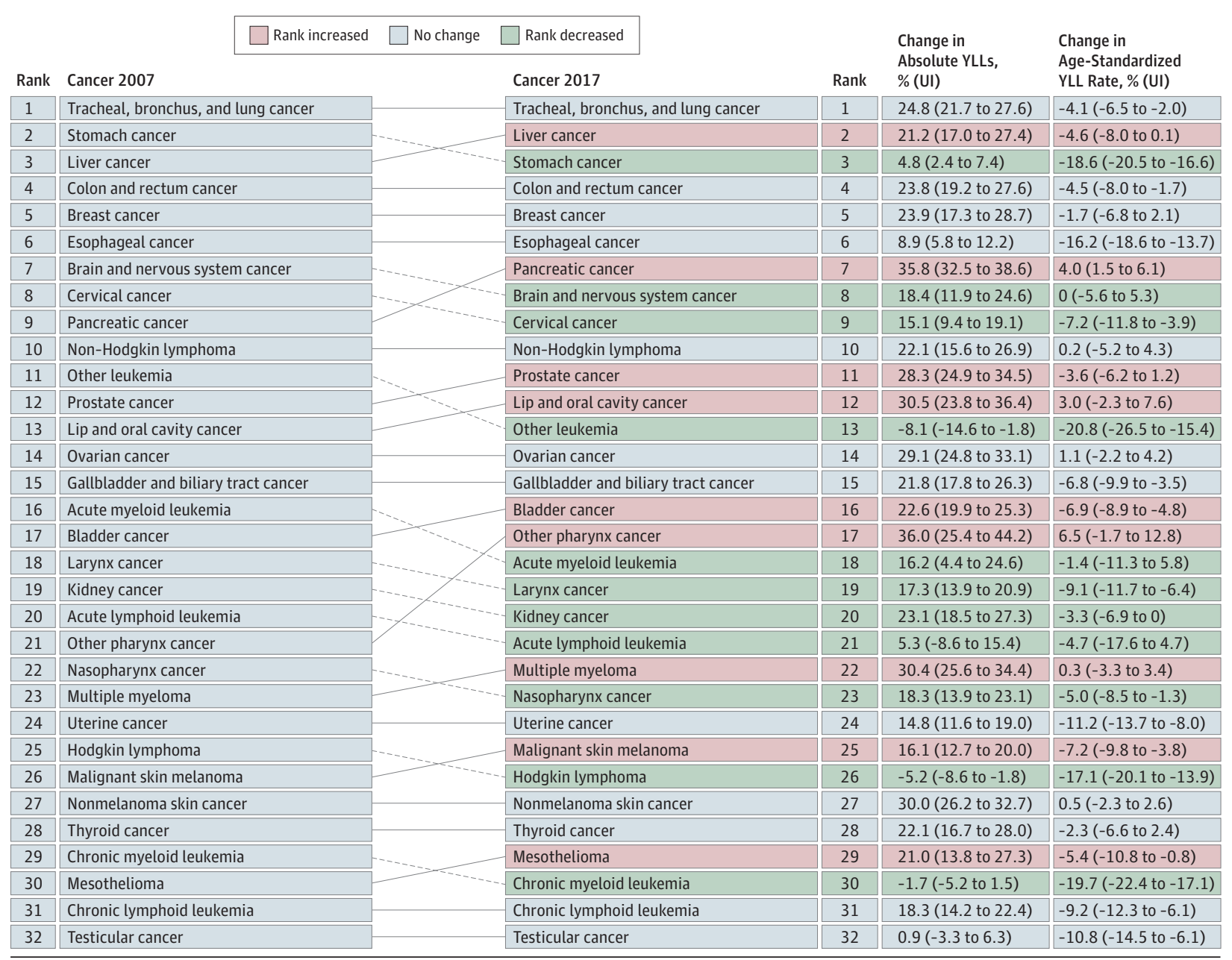

Ul indicates uncertainty interval.

${ }^{\text {a }}$ Excluding other cancer.

\section{Liver Cancer}

In 2017, there were 953000 (95\% UI, 917 000-997 000) incident cases of liver cancer globally and 819000 (95\% UI, 790 000-856 000) deaths. Liver cancer caused 20.8 million (95\% UI, 19.9-21.8 million) DALYs in 2017, with 99\% coming from YLLs and 1\% coming from YLDs (eTable 15 and eFigure 4 in the Supplement). Globally, liver cancer was more common in men, with 1 in 42 men developing liver cancer compared with 1 in 118 women. The highest odds of developing liver cancer were in high-middle SDI countries for men (1 in 31) and in middle SDI countries for women (1 in 94), whereas the lowest were seen in low SDI countries (1 in 98 men and 1 in 177 women) (eTable 16 in the Supplement). Population aging and population growth were the drivers of the increase from 705000 (95\% UI, 690 000-734 000) cases in 2007 to 953000 (95\% UI, 917 000-997 000) cases in 2017 (eTable 14 and eFigure 11 in the Supplement). Of the 35\% increase in cases between 2007 and $2017,17 \%$ was due to population aging, $13 \%$ due to population growth, and 6\% due to an increase in age-specific incidence rates.

\section{Cervical Cancer}

In 2017, 601000 (95\% UI, 554 000-625 000) women developed cervical cancer worldwide, and it caused 260000 (95\% UI, 241 000-269 000) deaths (Table). Cervical cancer caused 8.1 million (95\% UI, 7.5-8.4 million) DALYs, with 96\% coming from YLLs and 4\% from YLDs (eTable 15 and eFigure 4 in the Supplement). Globally, 1 in 65 women developed cervical cancer during a lifetime (eTable 16 in the Supplement). The odds were the highest in low SDI countries (1 in 40) and the lowest in high SDI countries (1 in 106). In 2017, cervical cancer was the most common incident cancer for women in 50 countries (eFigure 8 in the Supplement) and the most common cause of cancer deaths in 39 countries (eFigure 10 in the Supplement). Between 2007 and 2017, incident cases increased by 19\% (95\% UI, 13\%-23\%) globally. Population growth contributed $13 \%$ and population aging $9 \%$, while falling age-specific incidence rates offset this increase by $-3 \%$ (eFigure 11 and eTable 14 in the Supplement). Deaths increased by 19\% (95\% UI, 13\%-23\%) between 2007 and 2017, and DALYs by 15\% (95\% UI, 10\%-19\%). 
Figure 4. Change in the Absolute Number of Disability-Adjusted Life-Years (DALYs) Between 1990 and 2017 for Both Sexes at the Global Level for Global Burden of Disease Level 2 Causes $^{\text {a }}$

\begin{tabular}{|c|c|c|c|}
\hline \multirow[b]{2}{*}{ Rank } & \multirow[b]{2}{*}{ Cancer 2007} & \multirow[t]{2}{*}{$\square$ Noncommunicable diseases } & \multirow[t]{2}{*}{ juries } \\
\hline & & & \\
\hline 1 & Respiratory infections and tuberculosis & Cardiovascular diseases & 1 \\
\hline 2 & Maternal and neonatal diseases & Neoplasms & 2 \\
\hline 3 & Cardiovascular diseases & Maternal and neonatal disease & 3 \\
\hline 4 & Enteric infections & Respiratory infections and tuberculosis & 4 \\
\hline 5 & Other infections diseases & Musculoskeletal disorders & 5 \\
\hline 6 & Neoplasms & Mental disorders & 6 \\
\hline 7 & Other noncommunicable diseases & Other noncommunicable diseases & 7 \\
\hline 8 & Unintentional injury & Chronic respiratory disease & 8 \\
\hline 9 & Chronic respiratory disease & Neurological disorders & 9 \\
\hline 10 & Neglected tropical diseases and malaria & Unintentional injury & 10 \\
\hline 11 & Nutritional deficiencies & Diabetes and chronic kidney disease & 11 \\
\hline 12 & Musculoskeletal disorders & Enteric infections & 12 \\
\hline 13 & Mental disorders & Digestive diseases & 13 \\
\hline 14 & Transport injuries & Transport injuries & 14 \\
\hline 15 & Neurological disorders & Self-harm and violence & 15 \\
\hline 16 & Self-harm and violence & Sense organ diseases & 16 \\
\hline 17 & Digestive diseases & HIV/AIDS and sexually transmitted infections & 17 \\
\hline 18 & Diabetes and chronic kidney disease & Neglected tropical diseases and malaria & 18 \\
\hline 19 & Sense organ diseases & Nutritional deficiency & 19 \\
\hline 20 & HIV/AIDS and sexually transmitted infections & Other infectious diseases & 20 \\
\hline 21 & Skin diseases & Substance use & 21 \\
\hline 22 & Substance use & Skin diseases & 22 \\
\hline
\end{tabular}

The cause neoplasms includes all cancers as defined under International Statistical Classification of Diseases and Related Health Problems, Tenth Revision (ICD-10) causes COO through C96, as well as myelodysplastic, myeloproliferative, and other hematopoietic neoplasms (ICD-10 codes D45-D47.9)

${ }^{\text {a }}$ All diseases are grouped into 22 mutually exclusive and collectively exhaustive causes
The ASIRs decreased globally and for all SDI quintiles (eFigures 12-16 in the Supplement).

\section{Non-Hodgkin Lymphoma}

In 2017, there were 488000 (95\% UI, 479 000-497 000) incident cases of non-Hodgkin lymphoma and 249000 (95\% UI, 243 000-253 000) deaths. Non-Hodgkin lymphoma caused 7.0 million (95\% UI, 6.8-7.2 million) DALYs in 2017, with 97\% coming from YLLs and 3\% from YLDs (eTable 15 and eFigure 4 in the Supplement). Globally, 1 in 108 men and 1 in 162 women developed non-Hodgkin lymphoma over a lifetime. The highest odds were in high SDI countries (1 in 54 for men and 1 in 80 for women) and the lowest in low SDI countries (1 in 221 for men and 1 in 322 for women) (eTable 16 in the Supplement). Globally, incident cases between 2007 and 2017 increased by $39 \%$ (95\% UI, 35\%-42\%), of which $15 \%$ was due to changing population age structure, $13 \%$ due to population growth, and $11 \%$ due to change in incidence rates (eTable 14 and eFigure 11 in the Supplement).

\section{Bladder Cancer}

In 2017, there were 474000 (95\% UI, 462 000-492 000) incident cases of bladder cancer and 197000 (95\% UI, 192 000206000 ) deaths. Bladder cancer caused 3.6 million (95\% UI, 3.5-3.8 million) DALYs in 2017, with 93\% coming from YLLs and 7\% from YLDs (eTable 15 and eFigure 4 in the Supplement). Globally, 1 in 74 men and 1 in 301 women developed bladder cancer over a lifetime. The highest odds were in high SDI countries ( 1 in 42 for men and 1 in 185 for women) and the lowest in low SDI countries ( 1 in 198 for men and 1 in 489 for women)
(eTable 16 in the Supplement). Globally, incident cases between 2007 and 2017 increased by 32\% (95\% UI, 30\%-35\%), of which $20 \%$ was due to changing population age structure and $13 \%$ to population growth (eTable 14 and eFigure 11 in the Supplement).

\section{Cancer in Comparison to Other Diseases}

Within the 22 mutually exclusive and collectively exhaustive GBD level 2 disease categories (eTable 17 in the Supplement), neoplasms ranked last for incidence in 1990 and 2017 (eTable 18 in the Supplement). For prevalence, neoplasms ranked last in 1990 but surpassed enteric infections in 2017. The YLDs ranking for neoplasms also increased between 1990 and 2017 from the 21st to the 19th position. Mortality due to neoplasms remained at the second place between 1990 and 2017. The largest increase was seen for neoplasm YLLs and DALYs, which increased from the sixth place in 1990 to the second place in 2017 after cardiovascular diseases (Figure 4). The 4 causes with higher DALYs in 1990 that had been surpassed by neoplasms in 2017 are respiratory infections and tuberculosis, maternal and neonatal disorders, enteric infections, and other infections.

\section{Discussion}

The GBD study results are updated on an annual basis. In this article we focus on changes over the past decade and present the most recent results from the GBD 2017 study using cancer registry, vital registration, and verbal autopsy data to esti- 
mate the burden of cancer for 195 countries and territories from 1990 through $2017 . .^{13,14}$ All results presented can also be found online at https://vizhub.healthdata.org/gbd-compare/ and http://ghdx.healthdata.org/gbd-results-tool. For this article, we also compare cancer burden with other diseases.

The GBD 2017 results show that there are 24.5 million incident cancer cases worldwide (16.8 million without NMSC) and 9.6 million deaths, which is similar to the latest GLOBOCAN estimates for 2018 that estimate 17.0 million cases (without NMSC) and 9.4 million deaths. ${ }^{15}$

The largest change in our estimates compared with the last iteration of the GBD study (GBD 2016) are the incidence estimates for NMSC, which have substantially increased. Despite being the most common incident cancer in many populations, cancer registry data to inform incidence estimates are often unreliable or nonexistent. For GBD 2017 we have therefore used Marketscan data for the United States, which has led to substantially higher estimates for NMSC. ${ }^{16}$

A key strength of the GBD study is the comparative health assessment. Our analysis shows how cancer has increased in importance as a global health problem. Although it ranked sixth in 1990 among the top causes for DALYs worldwide, it has risen to the second place in 2017 behind cardiovascular diseases. Cancer now occupies the second place in the ranking of global deaths, YLLs, and DALYs, and is among the top 2 leading causes of deaths, YLLs, and DALYs in the highest 3 SDI quintiles. This shift in disease burden owing to the demographic and epidemiological transitions has important implications on health policy: ensuring access to universal health coverage and protection against catastrophic health expenditure directly related to the cancer treatment, but also against the long-term costs associated with a cancer diagnosis for a household, has to be prioritized. ${ }^{17}$ Fifty percent of cancer cases occur in high SDI countries, but only $30 \%$ of cancer deaths, $25 \%$ of cancer DALYs, and $23 \%$ of cancer YLLs. To ensure sustainable global development, increased efforts are needed to reduce these health inequalities. Recognizing the strong interdependencies between socioeconomic status and health and the large contribution of cancer to the overall disease burden is a first step in making investments in cancer prevention and treatment a priority. ${ }^{18}$ Cervical cancer is likely the best example of inequalities in cancer with vast differences in burden by SDI. As a completely preventable cancer where cost-effective vaccination $^{3}$ and screening approaches are available, cervical cancer has recently gained global attention through the World Health Organization's call for elimination. ${ }^{19}$ Falling incidence rates in all SDI quintiles are encouraging, but countries with the least resources are still facing the largest burden because of lack of screening programs. Immunization against human papillomavirus, screening, and treatment of cervical cancer is therefore of utmost importance in all socioeconomic settings.
Deaths due to cancer contribute the majority of total health loss measured in DALYs, with disability contributing less than $12 \%$ for all cancers. As access to cancer care increases and treatments improve, cancer mortality decreases, but prevalence and disability in the survivor population increase, which is already the case in some high-income countries. ${ }^{20}$ The World Health Organization Global Action Plan for the Prevention and Control of NCDs and the United Nations Sustainable Development Goals focus on the reduction of premature mortality as the first goal. At the same time, infrastructure should be planned that can address the growing survivor population's need.

\section{Limitations}

The most important limitation for the GBD, as for other disease burden estimation, is the lack of data for many locations. A key GBD principle is to take advantage of all relevant data sources. This means for cancer estimation that incidence data from cancer registries, as well as mortality data from vital registration systems or verbal autopsies, is used to produce disease burden estimates. Despite these broad inclusion criteria for different types of data sources, certain locations have neither of these data sources available, and estimates rely either on predictive covariates or trends from neighboring locations. Also, diagnostic accuracy for cause of death data and ascertainment bias in cancer registries remains a limitation, which requires corrections for underregistration and redistribution algorithms for insufficiently specific or implausible diagnostic codes. Because of a lag in data availability, estimates for the most recent years are based on past time trends and covariates rather than data, which is reflected in larger uncertainty. Scarcity of reliable survival data worldwide requires the estimation of survival based on the mortalityto-incidence ratio, which is a surrogate for survival. Because in the majority of deaths due to Kaposi sarcoma the underlying cause of deaths is AIDS, deaths and incidence of Kaposi sarcoma are not estimated in the GBD. Also, common pediatric cancers are not estimated separately in the GBD and are estimated under the aggregated cause "other malignant neoplasms.”

\section{Conclusions}

The national epidemiological profiles of cancer burden in the GBD study show large heterogeneities, which are a reflection of different exposures to risk factors, economic settings, lifestyles, and access to care. The GBD study can be used by policy makers and other stakeholders to develop and improve local cancer control in order to achieve the global targets and improve equity in cancer care.
ARTICLE INFORMATION

Accepted for Publication: April 30, 2019.

Published Online: September 27, 2019.

doi:10.1001/jamaoncol.2019.2996
Correction: This article was corrected on April 9 , 2020, to fix an error in a coauthor's affiliation. This article was also corrected on March 12, 2020, to fix errors in coauthors' names and an affiliation, Table data, Figure data, and the Supplement.
Open Access: This is an open access article distributed under the terms of the CC-BY License. (c) 2019 Global Burden of Disease Cancer Collaboration. JAMA Oncology. 
The Global Burden of Disease Cancer Collaboration Authors: The following investigators take authorship responsibility for the study results: Christina Fitzmaurice, MD; Degu Abate, MSc; Naghmeh Abbasi, MSc; Hedayat Abbastabar, PhD; Foad Abd-Allah, MD; Omar Abdel-Rahman, MD; Ahmed Abdelalim, MD; Amir Abdoli, PhD; Ibrahim Abdollahpour, PhD; Abdishakur S. M. Abdulle, PhD; Nebiyu Dereje Abebe, MPH; Haftom Niguse Abraha, MSc; Laith Jamal Abu-Raddad, PhD; Ahmed Abualhasan, MD; Isaac Akinkunmi Adedeji, PhD; Shailesh M. Advani, PhD; Mohsen Afarideh, MD; Mahdi Afshari, MD; Mohammad Aghaali, PhD; Dominic Agius, MD; Sutapa Agrawal, PhD; Ayat Ahmadi, PhD; Elham Ahmadian, PhD; Ehsan Ahmadpour, PhD; Muktar Beshir Ahmed, MPH; Mohammad Esmaeil Akbari, MD; Tomi Akinyemiju, PhD; Ziyad Al-Aly, MD; Assim M. AlAbdulKader, MD; Fares Alahdab, MD; Tahiya Alam, MPH; Genet Melak Alamene, BS; Birhan Tamene T. Alemnew, MSc; Kefyalew Addis Alene, MPH; Cyrus Alinia, PhD; Vahid Alipour, PhD; Syed Mohamed Aljunid, PhD; Fatemeh Allah Bakeshei, MPH; Majid Abdulrahman Hamad Almadi, FRCPC; Amir Almasi-Hashiani, PhD; Ubai Alsharif, MD; Shirina Alsowaidi, FRCPS; Nelson Alvis-Guzman, PhD; Erfan Amini, MD; Saeed Amini, PhD; Yaw Ampem Amoako, MD; Zohreh Anbari, PhD; Nahla Hamed Anber, PhD; Catalina Liliana Andrei, PhD; Mina Anjomshoa, PhD; Fereshteh Ansari, PhD; Ansariadi Ansariadi, PhD; Seth Christopher Yaw Appiah, MPhil; Morteza Arab-Zozani, PhD; Jalal Arabloo, PhD; Zohreh Arefi, PhD; Olatunde Aremu, PhD; Habtamu Abera Areri, MSc; Al Artaman, PhD; Hamid Asayesh, MSc; Ephrem Tsegay Asfaw, MSc; Alebachew Fasil Ashagre, MSc; Reza Assadi, PhD; Bahar Ataeinia, MD; Hagos Tasew Atalay, MSc; Zerihun Ataro, MSc; Suleman Atique, PhD; Marcel Ausloos, PhD; Leticia Avila-Burgos, PhD; Euripide F. G. A. Avokpaho, MD; Ashish Awasthi, PhD; Nefsu Awoke, MSc; Beatriz Paulina Ayala Quintanilla, PhD; Martin Amogre Ayanore, PhD; Henok Tadesse Ayele, PhD; Ebrahim Babaee, PhD; Umar Bacha, MPhil; Alaa Badawi, PhD; Mojtaba Bagherzadeh, PhD; Eleni Bagli, PhD; Senthilkumar Balakrishnan, PhD; Abbas Balouchi,

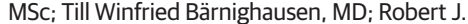
Battista, MBA; Masoud Behzadifar, PhD; Meysam Behzadifar, MS; Bayu Begashaw Bekele, MPH; Yared Belete Belay, MSc; Yaschilal Muche Belayneh, MSc; Kathleen Kim Sachiko Berfield, MD; Adugnaw Berhane, PhD; Eduardo Bernabe, PhD; Mircea Beuran, PhD; Nickhill Bhakta, MD; Krittika Bhattacharyya, MSc; Belete Biadgo, MSc; Ali Bijani, PhD; Muhammad Shahdaat Bin Sayeed, MSPS; Charles Birungi, MSc; Catherine Bisignano, MPH; Helen Bitew, MSc; Tone Bjørge, PhD; Archie Bleyer, MD; Kassawmar Angaw Bogale, MPH; Hunduma Amensisa Bojia, BPharm; Antonio M. Borzì, MD; Cristina Bosetti, PhD; Ibrahim R. Bou-Orm, MD; Hermann Brenner, MD; Jerry D. Brewer, MD; Andrey Nikolaevich Briko, MSc; Nikolay Ivanovich Briko, DSc; Maria Teresa Bustamante-Teixeira, PhD; Zahid A. Butt, PhD; Giulia Carreras, PhD; Juan J. Carrero, PhD; Félix Carvalho, PhD; Clara Castro, PhD; Franz Castro, MD; Ferrán Catalá-López, PhD; Ester Cerin, PhD; Yazan Chaiah; Wagaye Fentahun Chanie, MPH; Vijay Kumar Chattu, MD; Pankaj Chaturvedi, MD; Neelima Singh Chauhan, MD; Mohammad Chehrazi, PhD; Peggy Pei-Chia Chiang, PhD; Tesfaye Yitna Chichiabellu, MSc; Onyema Greg Chido-Amajuoyi, MD; Odgerel Chimed-Ochir, PhD; Jee-Young J. Choi, PhD; Devasahayam J. Christopher, MD; Dinh-Toi Chu, PhD;
Maria-Magdalena Constantin, MD; Vera M. Costa, PharmD; Emanuele Crocetti, MD; Christopher Stephen Crowe, MD; Maria Paula Curado, PhD; Saad M. A. Dahlawi, PhD; Giovanni Damiani, MD; Amira Hamed Darwish, MD; Ahmad Daryani, PhD; José das Neves, PhD; Feleke Mekonnen Demeke, MSc; Asmamaw Bizuneh Demis, MSc; Birhanu Wondimeneh Demissie, MSc; Gebre Teklemariam Demoz, MPharm; Edgar Denova-Gutiérrez, DSc; Afshin Derakhshani, MSc; Kalkidan Solomon Deribe, $\mathrm{MPH}$; Rupak Desai, MBBS; Beruk Berhanu Desalegn, MSc; Melaku Desta, MSc; Subhojit Dey, PhD; Samath Dhamminda Dharmaratne, MD; Meghnath Dhimal, PhD; Daniel Diaz, PhD; Mesfin Tadese Tadese Dinberu, MA; Shirin Djalalinia, PhD; David Teye Doku, PhD; Thomas M. Drake, MD; Manisha Dubey, PhD; Eleonora Dubljanin, PhD; Eyasu Ejeta Duken, MSc; Hedyeh Ebrahimi, MD; Andem Effiong, MB; Aziz Eftekhari, PhD; Iman El Sayed, PhD; Maysaa El Sayed Zaki, MD, PhD; Shaimaa I. El-Jaafary, MD; Ziad El-Khatib, PhD; Demelash Abewa Elemineh, MSc; Hajer Elkout, PhD; Richard G. Ellenbogen, MD; Aisha Elsharkawy, MD; Mohammad Hassan Emamian, PhD; Daniel Adane Endalew, MSc; Aman Yesuf Endries, MPH; Babak Eshrati, PhD; Ibtihal Fadhil, PhD; Vahid Fallah Omrani, PhD; Mahbobeh Faramarzi, PhD; Mahdieh Abbasalizad Farhangi, PhD; Andrea Farioli, PhD; Farshad Farzadfar, MD; Netsanet Fentahun, PhD; Eduarda Fernandes, PhD; Garumma Tolu Feyissa, PhD; Irina Filip, MD; Florian Fischer, PhD; James L. Fisher, PhD; Lisa M. Force, MD; Masoud Foroutan, PhD; Marisa Freitas, PhD; Takeshi Fukumoto, PhD; Neal D. Futran, MD; Silvano Gallus, DSc; Fortune Gbetoho Gankpe, MD; Reta Tsegaye Gayesa, MSc; Tsegaye Tewelde Gebrehiwot, MPH; Gebreamlak Gebremedhn Gebremeskel, MSc; Getnet Azeze Gedefaw, MSc; Belayneh K. Gelaw, MSc; Birhanu Geta, MSc; Sefonias Getachew, MPH; Kebede Embaye Gezae, MSc; Mansour Ghafourifard, PhD; Alireza Ghajar, MD; Ahmad Ghashghaee, BSc; Asadollah Gholamian, MSc; Paramjit Singh Gill, DM; Themba T. G. Ginindza, PhD; Alem Girmay, MSc; Muluken Gizaw, MPH; Ricardo Santiago Gomez, PhD; Sameer Vali Gopalani, MPH; Giuseppe Gorini, MD; Bárbara Niegia Garcia Goulart, DSc; Ayman Grada, MD; Maximiliano Ribeiro Guerra, PhD; Andre Luiz Sena Guimaraes, PhD; Prakash C. Gupta, DSc; Rahul Gupta, MD; Kishor Hadkhale, MPH; Arvin Haj-Mirzaian, MD; Arya Haj-Mirzaian, MD; Randah R. Hamadeh, DPhil; Samer Hamidi, DrPH; Lolemo Kelbiso Hanfore, MSc; Josep Maria Haro, MD; Milad Hasankhani, MSc; Amir Hasanzadeh, PhD; Hamid Yimam Hassen, MPH; Roderick J. Hay, MD; Simon I. Hay, FMedSci; Andualem Henok, MPH; Nathaniel J. Henry, BS; Claudiu Herteliu, PhD; Hagos D. Hidru, MPH; Chi Linh Hoang, BMedSc; Michael K. Hole, MD; Praveen Hoogar, PhD; Nobuyuki Horita, PhD; H. Dean Hosgood, PhD; Mostafa Hosseini, PhD; Mehdi Hosseinzadeh, PhD; Mihaela Hostiuc, PhD; Sorin Hostiuc, PhD; Mowafa Househ, PhD; Mohammedaman Mama Hussen, MA; Bogdan Ileanu, PhD; Milena D. llic, PhD; Kaire Innos, PhD; Seyed Sina Naghibi Irvani, MD; Kufre Robert Iseh, MD; Sheikh Mohammed Shariful Islam, PhD; Farhad Islami, PhD; Nader Jafari Balalami, PhD; Morteza Jafarinia, MSc; Leila Jahangiry, PhD; Mohammad Ali Jahani, PhD; Nader Jahanmehr, PhD; Mihajlo Jakovljevic, PhD; Spencer L. James, MD; Mehdi Javanbakht, PhD; Sudha Jayaraman, MD; Sun Ha Jee, PhD; Ensiyeh Jenabi, PhD; Ravi Prakash Jha, MSc; Jost B. Jonas, MD; Jitendra Jonnagaddala, PhD; Tamas Joo, MSc; Suresh Banayya Jungari, MA;
Mikk Jürisson, PhD; Ali Kabir, MD; Farin Kamangar, MD; André Karch, MD; Narges Karimi, MD; Ansar Karimian, DipLangStud; Amir Kasaeian, PhD; Gebremicheal Gebreslassie Kasahun, MSc; Belete Kassa, MSc; Tesfaye Dessale Kassa, MSc; Mesfin Wudu Kassaw, MSc; Anil Kaul, MD; Peter Njenga Keiyoro, PhD; Abraham Getachew Kelbore, MSc; Amene Abebe Kerbo, MPH; Yousef Saleh Khader, PhD; Maryam Khalilarjmandi, MSc; Ejaz Ahmad Khan, MPH; Gulfaraz Khan, PhD; Young-Ho Khang, MD; Khaled Khatab, PhD; Amir Khater, MD; Maryam Khayamzadeh, MD; Maryam Khazaee-Pool, PhD; Salman Khazaei, PhD; Abdullah T. Khoja, MD; Mohammad Hossein Khosravi, MD; Jagdish Khubchandani, PhD; Neda Kianipour, MA; Daniel Kim, DrPH; Yun Jin Kim, PhD; Adnan Kisa, PhD; Sezer Kisa, PhD; Katarzyna Kissimova-Skarbek, PhD; Hamidreza Komaki, MD; Ai Koyanagi, MD; Kristopher J. Krohn, MPH; Burcu Kucuk Bicer, BEP; Nuworza Kugbey, MSc; Vivek Kumar, MD; Desmond Kuupiel, MPH; Carlo La Vecchia, MD; Deepesh P. Lad, DM; Eyasu Alem Lake, BSc; Ayenew Molla Lakew, MPH; Dharmesh Kumar Lal, MD; Faris Hasan Lami, PhD; Qing Lan, PhD; Savita Lasrado, MS; Paolo Lauriola, MD; Jeffrey V. Lazarus, PhD; James Leigh, MD; Cheru Tesema Leshargie, MPH; Yu Liao, PhD; Miteku Andualem Limenih, MSc; Stefan Listl, PhD; Alan D. Lopez, PhD; Platon D. Lopukhov, PhD; Raimundas Lunevicius, PhD; Mohammed Madadin, MD; Sameh Magdeldin, PhD; Hassan Magdy Abd El Razek, MD; Azeem Majeed, MD; Afshin Maleki, PhD; Reza Malekzadeh, MD; Ali Manafi, MD; Navid Manafi, MD; Wondimu Ayele Manamo, MS; Morteza Mansourian, PhD; Mohammad Ali Mansournia, PhD; Lorenzo Giovanni Mantovani, DSc; Saman Maroufizadeh, PhD; Santi Martini S. Martini, DrPH; Tivani Phosa Mashamba-Thompson, PhD; Benjamin Ballard Massenburg, MD; Motswadi Titus Maswabi, PhD; Manu Raj Mathur, PhD; Colm McAlinden, PhD; Martin McKee, DSc; Hailemariam Abiy Alemu Meheretu, MPH; Ravi Mehrotra, PhD; Varshil Mehta, MD; Toni Meier, PhD; Yohannes A. Melaku, $\mathrm{MPH}$; Gebrekiros Gebremichael Meles, MPH; Hagazi Gebre Meles, MPH; Addisu Melese, MSc; Mulugeta Melku, MSc; Peter T. N. Memiah, DrPH; Walter Mendoza, MD; Ritesh G. Menezes, MD; Shahin Merat, MD; Tuomo J. Meretoja, MD; Tomislav Mestrovic, PhD; Bartosz Miazgowski, MD; Tomasz Miazgowski, MD; Kebadnew Mulatu M. Mihretie, MPH; Ted R. Miller, PhD; Edward J. Mills, PhD; Seyed Mostafa Mir, MSc; Hamed Mirzaei, PhD; Hamid Reza Mirzaei, PhD; Rashmi Mishra, BDS; Babak Moazen, MSc; Dara K. Mohammad, PhD Karzan Abdulmuhsin Mohammad, PhD; Yousef Mohammad, MD; Aso Mohammad Darwesh, PhD; Abolfazl Mohammadbeigi, PhD; Hiwa Mohammadi, PhD; Moslem Mohammadi, PhD; Mahdi

Mohammadian, BA; Abdollah

Mohammadian-Hafshejani, PhD; Milad

Mohammadoo-Khorasani, PhD; Reza

Mohammadpourhodki, MSc; Ammas Siraj Mohammed, BA; Jemal Abdu Mohammed, MPH; Shafiu Mohammed, PhD; Farnam Mohebi, MD; Ali H. Mokdad, PhD; Lorenzo Monasta, DSc; Yoshan Moodley, PhD; Mahmood Moosazadeh, PhD; Maryam Moossavi, PhD; Ghobad Moradi, PhD; Mohammad Moradi-Joo, MSc; Maziar Moradi-Lakeh, MD; Farhad Moradpour, PhD; Lidia Morawska, PhD; Joana Morgado-da-Costa, MSc; Naho Morisaki, MD; Shane Douglas Morrison, MD; Abbas Mosapour, PhD; Seyyed Meysam Mousavi, PhD; Achenef Asmamaw Muche, MPH; Oumer Sada S. Muhammed, MSc; Jonah Musa, MD; Ashraf F. 
Nabhan, PhD; Mehdi Naderi, MSc; Ahamarshan Jayaraman Nagarajan, MTech; Gabriele Nagel, PhD; Azin Nahvijou, PhD; Gurudatta Naik, MPH; Farid Najafi, PhD; Luigi Naldi, MD; Hae Sung Nam, PhD; Naser Nasiri, MSc; Javad Nazari, PhD; lonut Negoi, PhD; Subas Neupane, PhD; Polly A. Newcomb, PhD Haruna Asura Nggada, MD; Josephine W. Ngunjiri, DrPH; Cuong Tat Nguyen, MPH; Leila Nikniaz, PhD; Dina Nur Anggraini Ningrum, MPH; Yirga Legesse Nirayo, MS; Molly R. Nixon, PhD; Chukwudi A. Nnaji, MPH; Marzieh Nojomi, MD; Shirin Nosratnejad, PhD; Malihe Nourollahpour Shiadeh, PhD; Mohammed Suleiman Obsa, BHlthSci; Richard Ofori-Asenso, MSc; Felix Akpojene Ogbo, PhD; In-Hwan Oh, PhD; Andrew T. Olagunju, MD; Tinuke O. Olagunju, MD; Mojisola Morenike Oluwasanu, PhD; Abidemi E. Omonisi, FWACP; Obinna E. Onwujekwe, PhD; Anu Mary Oommen, MD; Eyal Oren, PhD; Doris D. V. Ortega-Altamirano, DrPH; Erika Ota, PhD; Stanislav S. Otstavnov, PhD; Mayowa Ojo Owolabi, DrM; Mahesh P A, DNB; Jagadish Rao Padubidri, MD; Smita Pakhale, MD; Amir H. Pakpour, PhD; Adrian Pana, MD; Eun-Kee Park, PhD; Hadi Parsian, PhD; Tahereh Pashaei, PhD; Shanti Patel, MD; Snehal T. Patil, MS; Alyssa Pennini, MSc; David M. Pereira, PhD; Cristiano Piccinelli, MPH; Julian David Pillay, PhD; Majid Pirestani, PhD; Farhad Pishgar, MD; Maarten J. Postma, PhD; Hadi Pourjafar, PhD; Farshad Pourmalek, PhD; Akram Pourshams, MD; Swayam Prakash, PhD; Narayan Prasad, MD; Mostafa Qorbani, PhD; Mohammad Rabiee, PhD; Navid Rabiee, PhD; Amir Radfar, MD; Alireza Rafiei, PhD Fakher Rahim, PhD; Mahdi Rahimi, PhD; Muhammad Aziz Rahman, PhD; Fatemeh Rajati, PhD; Saleem M. Rana, PhD; Samira Raoofi, MSc Goura Kishor Rath, MD; David Laith Rawaf, MD; Salman Rawaf, MD; Robert C. Reiner, PhD; Andre M. N. Renzaho, PhD; Nima Rezaei, PhD; Aziz Rezapour PhD; Ana Isabel Ribeiro, PhD; Daniela Ribeiro, PhD; Luca Ronfani, PhD; Elias Merdassa Roro, MPH; Gholamreza Roshandel, PhD; Ali Rostami, PhD; Ragy Safwat Saad, MSc; Parisa Sabbagh, PhD; Siamak Sabour, PhD; Basema Saddik, PhD; Saeid Safiri, PhD; Amirhossein Sahebkar, PhD; Mohammad Reza Salahshoor, PhD; Farkhonde Salehi, MA; Hosni Salem, MD; Marwa Rashad Salem, MD; Hamideh Salimzadeh, PhD; Joshua A.

Salomon, PhD; Abdallah M. Samy, PhD; Juan Sanabria, MD; Milena M. Santric Milicevic, PhD; Benn Sartorius, PhD; Arash Sarveazad, PhD; Brijesh Sathian, PhD; Maheswar Satpathy, PhD; Miloje Savic, PhD; Monika Sawhney, PhD; Mehdi Sayyah, MD; Ione J. C. Schneider, PhD; Ben Schöttker, PhD; Mario Sekerija, PhD; Sadaf G. Sepanlou, MD; Masood Sepehrimanesh, PhD; Seyedmojtaba Seyedmousavi, PhD; Faramarz Shaahmadi, PhD; Hosein Shabaninejad, PhD; Mohammad Shahbaz, MSc; Masood Ali Shaikh, MD; Amir Shamshirian, BMedSci; Morteza Shamsizadeh, MSc; Heidar Sharafi, PhD; Zeinab Sharafi, PhD; Mehdi Sharif, PhD; Ali Sharifi, MD; Hamid Sharifi, PhD; Rajesh Sharma, PhD; Aziz Sheikh, MD; Reza Shirkoohi, PhD; Sharvari Rahul Shukla, PhD; Si Si, PhD; Soraya Siabani, PhD; Diego Augusto Santos Silva, PhD; Dayane Gabriele Alves Silveira, MD; Ambrish Singh, MTech; Jasvinder A. Singh, MD; Solomon Sisay, $\mathrm{MPH}$; Freddy Sitas, PhD; Eugène Sobngwi, PhD Moslem Soofi, PhD; Joan B. Soriano, MD; Vasiliki Stathopoulou, PhD; Mu'awiyyah Babale Sufiyan, MD; Rafael Tabarés-Seisdedos, PhD; Takahiro Tabuchi, MD; Ken Takahashi, PhD; Omid Reza Tamtaji, MSc; Mohammed Rasoul Tarawneh, PhD;
Segen Gebremeskel Tassew, MSc; Parvaneh Taymoori, PhD; Arash Tehrani-Banihashemi, PhD; Mohamad-Hani Temsah, MD; Omar Temsah; Berhe Etsay Tesfay, MPH; Fisaha Haile Tesfay, MPH; Manaye Yihune Teshale, MPH; Gizachew Assefa Tessema, MPH; Subash Thapa, PhD; Kenean Getaneh Tlaye, MSc; Roman Topor-Madry, PhD; Marcos Roberto Tovani-Palone, MSc; Eugenio Traini, MSc; Bach Xuan Tran, PhD; Khanh Bao Tran, MD; Afewerki Gebremeskel Tsadik, MSc; Irfan Ullah, PhD; Olalekan A. Uthman, PhD; Marco Vacante, PhD; Maryam Vaezi, MD; Patricia Varona Pérez, PhD; Yousef Veisani, PhD; Simone Vidale, MD; Francesco S. Violante, MD; Vasily Vlassov, MD; Stein Emil Vollset, DrPH; Theo Vos, PhD; Kia Vosoughi, MD; Giang Thu Vu, BA; Isidora S. Vujcic, PhD; Henry Wabinga, MD; Tesfahun Mulatu Wachamo, MPH; Fasil Shiferaw Wagnew, MSc; Yasir Waheed, PhD; Fitsum Weldegebreal, MSc; Girmay Teklay Weldesamuel, MSc; Tissa Wijeratne, MD; Dawit Zewdu Wondafrash, MSc; Tewodros Eshete Wonde, $\mathrm{MPH} ;$ Adam Belay Wondmieneh, MSc; Hailemariam Mekonnen Workie, MSc; Rajaram Yadav, PhD; Abbas Yadegar, PhD; Ali Yadollahpour, PhD; Mehdi Yaseri, PhD; Vahid Yazdi-Feyzabadi, PhD; Alex Yeshaneh, BHIthSci; Mohammed Ahmed Yimam, $\mathrm{MPH}$; Ebrahim M. Yimer, MSc; Engida Yisma, MPH; Naohiro Yonemoto, MPH; Mustafa Z. Younis, PhD; Bahman Yousefi, PhD; Mahmoud Yousefifard, PhD Chuanhua Yu, PhD; Erfan Zabeh, BS; Vesna Zadnik، PhD; Telma Zahirian Moghadam, PhD; Zoubida Zaidi, PhD; Mohammad Zamani, MD; Hamed Zandian, PhD; Alireza Zangeneh, MD; Leila Zaki, PhD; Kazem Zendehdel, PhD; Zerihun Menlkalew Zenebe, MSc; Taye Abuhay Zewale, MSc; Arash Ziapour, PhD; Sanjay Zodpey, PhD; Christopher J. L. Murray, DPhil.

\section{Affiliations of The Global Burden of Disease} Cancer Collaboration Authors: Institute for Health Metrics and Evaluation, University of Washington, Seattle, (Fitzmaurice, Alam, Bisignano, Dharmaratne, Force, S. I. Hay, Henry, James, Krohn, Lopez, Mokdad, Nixon, Pennini, Reiner, Vollset, Vos, Murray); Division of Hematology, University of Washington, Seattle (Fitzmaurice); Haramaya University, Harar, Ethiopia (Abate); Department of Clinical Biochemistry, Babol University of Medical Sciences, Babol, Iran (Abbasi, Khalilarjmandi, Mir, Mosapour, Parsian); Iranian Center of Neurological Research, Tehran University of Medical Sciences, Tehran, Iran (Abbastabar); Department of Neurology, Cairo University, Cairo, Egypt (Abd-Allah, Abdelalim, Abualhasan, El-Jaafary); Department of Oncology, University of Calgary, Calgary, Alberta, Canada (Abdel-Rahman); Department of Oncology, Ain Shams University, Cairo, Egypt (Abdel-Rahman); Department of Parasitology and Mycology, Jahrom University of Medical Sciences, Jahrom, Iran (Abdoli); Research Center for Non-communicable Diseases, Jahrom University of Medical Sciences, Jahrom, Iran (Abdoli); Department of Epidemiology, Arak University of Medical Sciences, Arak, Iran (Abdollahpour, Almasi-Hashiani); Multiple Sclerosis Research Center, Tehran, Iran (Abdollahpour); Public Health Research Center, New York University Abu Dhabi, Abu Dhabi, United Arab Emirates (Abdulle); School of Public Health, Addis Ababa University, Addis Ababa, Ethiopia (Abebe, Berhane, Gizaw, Manamo, Roro); Department of Public Health, Wachemo University, Hossana, Ethiopia (Abebe); Clinical Pharmacy Unit, Mekelle University, Mekelle, Ethiopia (Abraha, T. D. Kassa, Nirayo);
Department of Healthcare Policy and Research, Weill Cornell Medical College in Qatar, Doha, Qatar (Abu-Raddad); Department of Sociology, Olabisi Onabanjo University, Ago Iwoye, Nigeria (Adedeji); Social Behavioral Research Branch, National Institutes of Health, Bethesda, Maryland (Advani); Cancer Prevention and Control Program, Georgetown University, Washington, DC (Advani); Endocrinology and Metabolism Research Institute, Tehran University of Medical Sciences, Tehran, Iran (Afarideh, Ghajar, Rahim); Zabol University of Medical Sciences, Zabol, Iran (Afshari); Department of Epidemiology and Biostatistics, Qom University of Medical Sciences, Qom, Iran (Aghaali, Mohammadbeigi); Department of Health, Directorate for Health Information and Research, Pieta, Malta (Agius); Public Health Foundation of India, Gurugram, India (Agrawal, Awasthi, Lal, Mathur, Zodpey); Vital Strategies, Gurugram, India (Agrawal); Knowledge Utilization Research Center, Tehran University of Medical Sciences, Tehran, Iran (Ahmadi); Department of Pharmacology and Toxicology, Tabriz University of Medical Sciences, Tabriz, Iran (Ahmadian, Eftekhari); Department of Parasitology and Mycology, Tabriz University of Medical Sciences, Tabriz, Iran (Ahmadpour); Department of Epidemiology, Jimma University, Jimma, Ethiopia (Ahmed, Gebrehiwot); Cancer Research Center, Shahid Beheshti University of Medical Sciences, Tehran, Iran (Akbari, Khayamzadeh); Department of Population Health Sciences, Duke University, Durham, North Carolina (Akinyemiju); Duke Global Health Institute, Duke University, Durham, North Carolina (Akinyemiju); John T. Milliken Department of Internal Medicine, Washington University in St. Louis, St Louis, Missouri (Al-Aly); Clinical Epidemiology Center, VA Saint Louis Health Care System, Department of Veterans Affairs, St Louis, Missouri (Al-Aly); Department of Family and Community Medicine, Imam Abdulrahman Bin Faisal University, Dammam, Saudi Arabia (AlAbdulKader); Department of Family Medicine and Community Health, Case Western Reserve University, Cleveland, Ohio (AlAbdulKader); Evidence-Based Practice Research Center, Mayo Clinic Foundation for Medical Education and Research, Rochester, Minnesota (Alahdab); School of Health Sciences, Madda Walabu University, Bale Goba, Ethiopia (Alamene); Department of Health Sciences, Woldia University, Woldia, Ethiopia (Alemnew); Department of Microbiology, Immunology, and Parasitology, Addis Ababa University, Addis Ababa, Ethiopia (Alemnew); Institute of Public Health, University of Gondar, Gondar, Ethiopia (Alene, Bekele, Limenih, Melaku, Melku, Muche, Tessema); Research School of Population Health, Australian National University, Canberra, Australian Capitol Territory, Australia (Alene); Department of Health Care Management and Economics, Urmia University of Medical Science, Urmia, Iran (Alinia); Health Management and Economics Research Center, Iran University of Medical Sciences, Tehran, Iran (Alipour, Arabloo, Masoud Behzadifar); Department of Health Economics, Iran University of Medical Sciences, Tehran, Iran (Alipour); Department of Health Policy and Management, Kuwait University, Safat, Kuwait (Aljunid); International Centre for Casemix and Clinical Coding, National University of Malaysia, Bandar Tun Razak, Malaysia (Aljunid); Department of Social Medicine, Behbahan Faculty of Medical Sciences, Behbahan, Iran (Bakeshei); Department of Medicine, King Saud University, 
Riyadh, Saudi Arabia (Almadi); Department of Gastroenterology and Hepatology, McGill University, Montreal, Québec, Canada (Almadi); Department of Oral and Maxillofacial Surgery, University Hospital Knappschaftskrankenhaus Bochum, Bochum, Germany (Alsharif); College of Medicine and Health Sciences, United Arab Emirates University, Al-Ain, United Arab Emirates (Alsowaidi); Research Group in Health Economics, Universidad de Cartagena, Cartagena, Colombia (Alvis-Guzman); Research Group in Hospital Management and Health Policies, Universidad de la Costa, Barranquilla, Colombia (Alvis-Guzman); Department of Urology, Tehran University of Medical Sciences, Tehran, Iran (E. Amini); Department of Health Services Management, Arak University of Medical Sciences, Arak, Iran (S. Amini, Anbari); Department of Internal Medicine, Komfo Anokye Teaching Hospital, Kumasi, Ghana (Amoako); Mansoura University, Mansoura, Egypt (Anber); Carol Davila University of Medicine and Pharmacy, Bucharest, Romania (Andrei); Social Determinants of Health Research Center, Rafsanjan University of Medical Sciences, Rafsanjan, Iran (Anjomshoa); Research Center for Evidence Based Medicine, Health Management and Safety Promotion Research Institute, Tabriz University of Medical Sciences, Tabriz, Iran (Ansari); School of Public Health, Hasanuddin University, Makassar, Indonesia (Ansariadi); Department of Sociology and Social Work, Kwame Nkrumah University of Science and Technology, Kumasi, Ghana (Appiah); Center for International Health, Ludwig Maximilians University, Munich, Germany (Appiah); Department of Healthcare Management, Tabriz University of Medical Sciences, Tabriz, Iran (Arab-Zozani); Department of Health Education and Health Promotion, Tehran University of Medical Sciences Tehran, Iran (Arefi); School of Health Sciences, Birmingham City University, Birmingham, England, United Kingdom (Aremu); School of Nursing and Midwifery, Addis Ababa University, Addis Ababa, Ethiopia (Areri); Department of Community Health Sciences, University of Manitoba, Winnipeg, Manitoba, Canada (Artaman); Qom University of Medical Sciences, Qom, Iran (Asayesh); Institute of Biomedical Science, Mekelle University, Mekelle, Ethiopia (Asfaw); Department of Clinical Chemistry, University of Gondar, Gondar, Ethiopia (Ashagre, Biadgo); Education Development Center, Mashhad University of Medical Sciences, Mashhad, Iran (Assadi); Non-communicable Diseases Research Center, Tehran University of Medical Sciences, Tehran, Iran (Ataeinia, Ebrahimi, Farzadfar, Irvani, Mohebi, Pishgar); College of Nursing, Aksum University, Aksum, Ethiopia (Atalay, Gebremeskel, Weldesamuel); Department of Medical Laboratory Science, Haramaya University, Harar, Ethiopia (Ataro, Weldegebreal); University Institute of Public Health, The University of Lahore, Lahore, Pakistan (Atique); College of Public Health, University of Hail, Hail, Saudi Arabia (Atique); School of Business, University of Leicester, Leicester, England, United Kingdom (Ausloos); Center for Health Systems Research, National Institute of Public Health, Cuernavaca, Mexico (Avila-Burgos, Ortega-Altamirano); Bénin Clinical Research Institute, Abomey-Calavi, Benin (Avokpaho); Contrôle des Maladies Infectieuses, Laboratory of Studies and Research-Action in Health, Porto Novo, Benin (Avokpaho); Indian Institute of Public Health, Gandhinagar, India (Awasthi); Department of Nursing, Wolaita Sodo University, Sodo, Ethiopia
(Awoke, Chichiabellu, Demissie, Hanfore, Lake); The Judith Lumley Centre, La Trobe University, Melbourne, Victoria, Australia (Ayala Quintanilla); General Office for Research and Technological Transfer, Peruvian National Institute of Health, Lima, Peru (Ayala Quintanilla); Department of Family and Community Health, School of Public Health, University of Health and Allied Sciences, Ho, Ghana (Ayanore, Kugbey); Department of Epidemiology, Biostatistics, and Occupational Health, McGill University, Montreal, Québec, Canada (Ayele); Public Health Department, Dilla University, Dilla, Ethiopia (Ayele); Preventive Medicine and Public Health Research Center, Iran University of Medical Sciences, Tehran, Iran (Babaee, Moradi-Lakeh, Nojomi,

Tehrani-Banihashemi); School of Health Sciences, University of Management and Technology, Lahore, Pakistan (Bacha); Public Health Risk Sciences Division, Public Health Agency of Canada, Toronto, Ontario, Canada (Badawi); Department of Nutritional Sciences, University of Toronto, Toronto, Ontario, Canada (Badawi); Department of Chemistry, Sharif University of Technology, Tehran, Iran (Bagherzadeh, N. Rabiee); Department of Ophthalmology, University Hospital of Ioannina, Ioannina, Greece (Bagli); Institute of Molecular Biology \& Biotechnology, Foundation for Research \& Technology, loannina, Greece (Bagli); Department of Medical Microbiology, Haramaya University, Harar, Ethiopia (Balakrishnan); School of Nursing and Allied Medicine, Iran University of Medical Sciences, Tehran, Iran (Balouchi); Heidelberg Institute of Global Health, Faculty of Medicine and University Hospital, Heidelberg University, Heidelberg, Germany (Bärnighausen); Harvard T. H. Chan School of Public Health, Harvard University, Boston, Massachusetts (Bärnighausen, P. C. Gupta); Doctor Evidence, Santa Monica, California (Battista); Social Determinants of Health Research Center, Lorestan University of Medical Sciences, Khorramabad, Iran (Masoud Behzadifar); Lorestan University of Medical Sciences, Khorramabad, Iran (Meysam Behzadifar); Public Health Department, Mizan-Tepi University, Teppi, Ethiopia (Bekele, Hassen); Department of Pharmacoepidemiology and Social Pharmacy, Mekelle University, Mekelle, Ethiopia (Belay); AC Environments Foundation, Cuernavaca, Mexico (Belay); Department of Pharmacy, Wollo University, Dessie, Ethiopia (Belayneh, Geta); Division of Cardiothoracic Surgery, University of Washington, Seattle, (Berfield); Dental Institute, King's College London, London, England, United Kingdom (Bernabe); Emergency Hospital of Bucharest, Carol Davila University of Medicine and Pharmacy, Bucharest, Romania (Beuran, Negoi); Department of Global Pediatric Medicine, St. Jude Children's Research Hospital, Memphis, Tennessee (Bhakta); Department of Biostatistics and Bioinformatics, National Institute of Biomedical Genomics, Kalyani, India (Bhattacharyya); Social Determinants of Health Research Center, Babol University of Medical Sciences, Babol, Iran (Bijani); National Centre for Epidemiology \& Population Health, Australian National University, Canberra, Australian Capital Territory, Australia (Bin Sayeed): Department of Clinical Pharmacy and Pharmacology, University of Dhaka, Ramna, Bangladesh (Bin Sayeed); The UCL Centre for Global Health Economics, University College London, London, England, United Kingdom (Birungi); Fast-Track Implementation Department.
United Nations Programme on HIV/AIDS,

Gaborone, Botswana (Birungi); School of Pharmacy, Mekelle University, Mekelle, Ethiopia (Bitew, Tsadik Yimer); Department of Global Public Health and Primary Care, University of Bergen, Bergen, Norway (Bjørge); Cancer Registry of Norway, Oslo, Norway (Bjørge); Department of Radiation Medicine, Oregon Health and Science University, Portland (Bleyer); Department of Pediatrics, The University of Texas, Houston (Bleyer); Department of Public Health and Epidemiology, Bahir Dar University, Bahir Dar, Ethiopia (Bogale); School of Pharmacy, College of Medicine and Health Science, Haramaya University, Harar, Ethiopia (Bojia, A. S. Mohammed) Department of Clinical and Molecular Biomedicine, University of Catania, Catania, Italy (Borzi); Department of Oncology, Mario Negri Institute for Pharmacological Research, Milan, Italy (Bosetti); Ministry of Public Health, Beirut, Lebanon (Bou-Orm); Division of Clinical Epidemiology and Aging Research, German Cancer Research Center, Heidelberg, Germany (Brenner, Schöttker): Department of Dermatology, Mayo Clinic, Rochester, Minnesota (Brewer); Biomedical Technologies, Bauman Moscow State Technical University, Moscow, Russia (A. N. Briko); Department of Epidemiology and Evidence-Based Medicine, I. M. Sechenov First Moscow State Medical University, Moscow, Russia (N. I. Briko, Lopukhov); Public Health Department, Federal University of Juiz de Fora, Juiz de Fora, Brazil (Bustamante-Teixeira); School of Population and Public Health, University of British Columbia, Vancouver, British Columbia, Canada (Butt, Pourmalek); Al Shifa School of Public Health, Al Shifa Trust Eye Hospital, Rawalpindi, Pakistan (Butt); Institute for Cancer Research, Prevention and Clinical Network, Florence, Italy (Carreras); Department of Medical Epidemiology and Biostatistics, Karolinska Institutet, Stockholm, Sweden (Carrero); Applied Molecular Biosciences Unit, University of Porto, Porto, Portugal (Carvalho); Institute of Public Health, University of Porto, Porto, Portugal (Carvalho); Department of Epidemiology, Portuguese Oncology Institute of Porto, Porto, Portugal (C. Castro); EpiUnit, Instituto de Saúde Pública, University of Cartagena, Cartagena, Colombia (C. Castro); Department of Research and Health Technology Assessment, Gorgas Memorial Institute for Health Studies, Panama City, Panama (F. Castro); National School of Public Health, Carlos III Health Institute, Madrid, Spain (Catalá-López); Clinical Epidemiology Program, Ottawa Hospital Research Institute, Ottawa, Ontario, Canada (Catalá-López); Mary MacKillop Institute for Health Research, Australian Catholic University, Melbourne, Victoria, Australia (Cerin); School of Public Health, The University of Hong Kong, Hong Kong, China (Cerin); College of Medicine, Alfaisal University, Riyadh, Saudi Arabia (Chaiah, M. Temsah, O. Temsah); Department of Obstetrics and Gynecology, University of Gondar, Gondar, Ethiopia (Chanie); Department of Psychiatry, University of Toronto, Toronto, Ontario, Canada (Chattu); China Institute, University of Alberta, Edmonton, Alberta, Canada (Chattu); Department of Surgical Oncology, Tata Memorial Hospital, Mumbai, India (Chaturvedi); Department of Obstetrics and Gynecology, People's College of Medical Sciences and Research Centre, Bhopal, India (Chauhan); Department of Biostatistics and Epidemiology, Babol University of Medical Sciences, Babol, Iran (Chehrazi); Epidemiology Research 
Center, Royan Institute, Tehran, Iran (Chehrazi); Clinical Governance, Gold Coast Health, Gold Coast, Queensland, Australia (Chiang); Department of Epidemiology, Human Genetics, and Environmental Sciences, The University of Texas, Houston (Chido-Amajuoyi); Institute of Industrial Ecological Science, University of Occupational and Environmental Health, Kitakyushu, Japan (Chimed-Ochir); Departments of Biochemistry and Biomedical Science, Seoul National University Hospital, Seoul, South Korea (Choi); Department of Pulmonary Medicine, Christian Medical College and Hospital, Vellore, India (Christopher); Faculty of Biology, Hanoi National University of Education, Hanoi, Vietnam (Chu); Department of Cancer Immunology, Oslo University Hospital, Oslo, Norway (Chu); Department of Dermatology, 2nd Clinic of Dermatology, Carol Davila University of Medicine and Pharmacy, Bucharest, Romania (Constantin); 2nd Department of Dermatology, Colentina Clinical Hospital, Bucharest, Romania (Constantin); UCIBIO/REQUIMTE, Laboratory of Toxicology, Faculty of Pharmacy, University of Porto, Porto, Portugal (Costa); Istituto Scientifico Romagnolo per lo Studio e la Cura dei Tumori (IRST) IRCCS, Meldola, Italy (Crocetti); Division of Plastic Surgery, University of Washington, Seattle (Crowe, Massenburg); Department of Epidemiology, A. C. Camargo Cancer Center, Sao Paulo, Brazil (Curado): Department of Environmental Health, College of Public Health, Imam Abdulrahman Bin Faisal University, Dammam, Saudi Arabia (Dahlawi); Department of Dermatology, Case Western Reserve University, Cleveland, Ohio (Damiani); Pediatric Department, Faculty of Medicine, Tanta University, Tanta, Egypt (Darwish); Toxoplasmosis Research Center, Mazandaran University of Medical Sciences, Sari, Iran (Daryani); Institute for Research and Innovation in Health (i3S), University of Porto, Porto, Portugal (das Neves); Institute of Biomedical Engineering (INEB), University of Porto, Porto, Portugal (das Neves); Department of Medical Laboratory Sciences, College of Medicine and Health Sciences, Bahir Dar University, Bahir Dar, Ethiopia (Demeke); Nursing Department, Woldia University, Woldia, Ethiopia (Demis, Kassaw, Tlaye); Department of Nursing, Jimma University, Jimma, Ethiopia (Demis); School of Pharmacy, Aksum University, Aksum, Ethiopia (Demoz, Kasahun); Addis Ababa University, Addis Ababa, Ethiopia (Demoz); Center for Nutrition and Health Research, National Institute of Public Health, Cuernavaca, Mexico (Denova-Gutiérrez); Department of Immunology, Birjand University of Medical Sciences, Birjand, Iran (Derakhshani); Department of Preventive Medicine, Addis Ababa University, Addis Ababa, Ethiopia (Deribe, Getachew); Division of Cardiology, Atlanta Veterans Affairs Medical Center, Decatur, Georgia (Desai); School of Nutrition, Food Science and Technology, Hawassa University, Hawassa, Ethiopia (Desalegn); Department of Midwifery, Debre Berhan University, Debre Berhan, Ethiopia (Desta, Dinberu); Faculty of Veterinary Medicine and Zootechnics, Autonomous University of Sinaloa, Culiacán Rosales, Mexico (Desta, Diaz); Disha Foundation, Gurgaon, India (Dey); Department of Community Medicine, University of Peradeniya, Peradeniya, Sri Lanka (Dharmaratne); Health Research Section, Nepa Health Research Council, Kathmandu, Nepal (Dhimal); Center of Complexity Sciences, Nationa Autonomous University of Mexico, Mexico City, Mexico (Diaz); Research and Technology, Ministry of Health and Medical Education, Tehran, Iran (Djalalinia); Department of Population and Health, University of Cape Coast, Cape Coast, Ghana (Doku); Faculty of Social Sciences, Health Sciences University of Tampere, Tampere, Finland (Doku) Department of Clinical Surgery, University of Edinburgh, Edinburgh, Scotland, United Kingdom (Drake); United Nations World Food Programme, New Delhi, India (Dubey); Faculty of Medicine, University of Belgrade, Belgrade, Serbia (Dubljanin, Vujcic); College of Health Sciences, Wollega University, Nekemte, Ethiopia (Duken); Mycobacteriology Research Center, Jimma University, Jimma, Ethiopia (Duken); Liver and Pancreaticobiliary Disease Research Center, Tehran University of Medical Sciences, Tehran, Iran (Ebrahimi); Department of Clinical Epidemiology and Biostatistics, University of Newcastle, Newcastle, New South Wales, Australia (Effiong); Department of Basic Sciences, Maragheh University of Medical Sciences, Maragheh, Iran (Eftekhari); Medical Research Institute, Alexandria University, Alexandria, Egypt (El Sayed); Department of Clinical Pathology, Mansoura University, Mansoura, Egypt (M. E. S. Zaki); Department of Public Health Sciences, Karolinska Institutet, Stockholm, Sweden (El-Khatib); Department of Statistics, Debre Markos University, Debre Markos, Ethiopia (Elemineh); Department of Community Medicine, Tripoli University, Tripoli, Libya (Elkout); Department of Health Information, World Health Organization, Tripoli, Libya (Elkout); Department of Neurology, University of Washington, Seattle (Ellenbogen); Department of Surgery, Seattle Children's Hospital Seattle, Washington (Ellenbogen); Endemic Medicine and Hepatogastroenterology Department, Cairo University, Cairo, Egypt (Elsharkawy); Ophthalmic Epidemiology Research Center, Shahroud University of Medical Sciences, Shahroud, Iran (Emamian); Department of Midwifery, Wolkite University, Wolkite, Ethiopia (Endalew); Public Health Department, St Paul's Hospital Millennium Medical College, Addis Ababa, Ethiopia (Endries); Center of Communicable Disease Control, Ministry of Health and Medical Education, Tehran, Iran (Eshrati); School of Public Health, Arak University of Medical Sciences, Arak, Iran (Eshrati); Department of Non-communicable Diseases, Ministry of Public Health, Dubai, United Arab Emirates (Fadhil); Shahid Beheshti University of Medical Sciences, Tehran, Iran (Fallah Omrani); Babol University of Medical Sciences, Babol, Iran (Faramarzi); Department of Community Nutrition, Tabriz University of Medical Sciences, Tabriz, Iran (Farhangi); Department of Medical and Surgical Sciences, University of Bologna, Bologna, Italy (Farioli, Violante); Department of Public Health Nutrition, Bahir Dar University, Bahir Dar, Ethiopia (Fentahun); Requimte/LAQV, University of Porto, Porto, Portugal (Fernandes, Pereira); Department of Health Education and Behavioral Sciences, Jimma University, Jimma, Ethiopia (Feyissa); Jimma University, Jimma, Ethiopia (Feyissa); Department of Psychiatry, Kaiser Permanente, Fontana, California (Filip); School of Health Sciences, A.T. Still University, Mesa, Arizona (Filip); School of Public Health Medicine, Bielefeld University, Bielefeld, Germany (Fischer); James Cancer Hospital, Ohio State University, Columbus, Ohio (Fisher); Department of Oncology, St. Jude Children's Research Hospital, Memphis, Tennessee (Force): Abadan School of Medical Sciences, Abadan, Iran (Foroutan); Department of Chemical Sciences,
Faculty of Pharmacy, University of Porto, Porto, Portugal (Freitas); Gene Expression and Regulation Program, Cancer Institute, Philadelphia, Pennsylvania (Fukumoto); Department of Dermatology, Kobe University, Kobe, Japan (Fukumoto); Department of Otolaryngology-Head and Neck Surgery, University of Washington, Seattle (Futran); Department of Environmental Health Science, Mario Negri Institute for Pharmacological Research, Milan, Italy (Gallus); Faculty of Medicine and Pharmacy of Fez, University Sidi Mohammed Ben Abdellah, Fez, Morocco (Gankpe); Non-communicable Disease Department, Laboratory of Studies and Research-Action in Health, Porto Novo, Benin (Gankpe); Department of Nursing, Wollega University, Nekemte, Ethiopia (Gayesa); Department of Nursing, Mekelle University, Mekelle, Ethiopia (Gebremeskel); Bahir Dar University, Bahir Dar, Ethiopia (Gedefaw); Haramaya University, Dire Dawa, Ethiopia (Gedefaw); School of Pharmacy, Ambo University, Ambo, Ethiopia (Gelaw); Institute of Epidemiology, Biostatistics and Informatics, Martin Luther University Halle-Wittenberg, Halle, Germany (Getachew); Department of Biostatistics, Mekelle University, Mekelle, Ethiopia (Gezae); Medical Surgical Department, Tabriz University of Medical Sciences, Tabriz, Iran (Ghafourifard); Department of Medicine, Massachusetts General Hospital, Boston (Ghajar); Department of Health Services Management, School of Health Management and Information Sciences, Iran University of Medical Sciences, Tehran, Iran (Ghashghaee, Raoofi); Physiology Department, Iran University of Medical Sciences, Tehran, Iran (Gholamian); Medical Department, Islamic Azad University, Rasht, Iran (Gholamian); Unit of Academic Primary Care, University of Warwick, Coventry, England, United Kingdom (Gill); Department of Public Health Medicine, University of KwaZulu-Natal, Durban, South Africa (Ginindza, Kuupiel, Mashamba-Thompson, Moodley); University of KwaZulu-Natal, Durban, South Africa (Ginindza); Department of Nursing, A.C.S. Medical College and Hospital, Aksum, Ethiopia (Girmay); Department of Surgery, Federal University of Minas Gerais, Belo Horizonte, Brazil (Gomez); Department of Biostatistics and Epidemiology, University of Oklahoma, Oklahoma City (Gopalani); Department of Health and Social Affairs, Government of the Federated States of Micronesia, Palikir, Federated States of Micronesia (Gopalani); Occupational and Environmental Epidemiology Section, Cancer Prevention and Research Institute, Florence, Italy (Gorini); Postgraduate Program in Epidemiology, Federal University of Rio Grande do Sul, Porto Alegre, Brazil (Goulart); School of Medicine, Boston University, Boston, Massachusetts (Grada); Department of Public Health, Federal University of Juiz de Fora, Juiz de Fora, Brazil (Ribeiro Guerra); School of Dentistry, State University of Montes Claros, Montes Claros, Brazil (Guimaraes); Department of Epidemiology, Healis Sekhsaria Institute for Public Health, Mumbai, India (P. C. Gupta); West Virginia Bureau for Public Health, Charleston (R. Gupta); Department of Health Policy, Management \& Leadership, West Virginia University, Morgantown (R. Gupta); University of Tampere, UKK Institute, Tampere, Finland (Hadkhale); Department of Pharmacology, Tehran University of Medical Sciences, Tehran, Iran (Arvin Haj-Mirzaian, Arya Haj-Mirzaian); Obesity Research 
Center, Shahid Beheshti University of Medical Sciences, Tehran, Iran (Arvin Haj-Mirzaian); Department of Radiology, Johns Hopkins University, Baltimore, Maryland (Arya Haj-Mirzaian); Department of Family and Community Medicine, Arabian Gulf University, Manama, Bahrain (Hamadeh); School of Health and Environmental Studies, Hamdan Bin Mohammed Smart University, Dubai, United Arab Emirates (Hamidi); Biomedical Research Networking Center for Mental Health Network, Madrid, Spain (Haro); Research and Development Unit, San Juan de Dios Sanitary Park, Sant Boi de Llobregat, Spain (Haro); School of Nutrition and Food Sciences, Tabriz University of Medical Sciences, Tabriz, Iran (Hasankhani); Department of Microbiology, Maragheh University of Medical Sciences, Maragheh, Iran (Hasanzadeh); Department of Microbiology, Tehran University of Medical Sciences, Tehran, Iran (Hasanzadeh); Unit of Epidemiology and Social Medicine, University Hospital Antwerp, Wilrijk, Belgium (Hassen); International Foundation for Dermatology, London, England, United Kingdom (R. J. Hay); St John's Institute of Dermatology, King's College London, London, England, United Kingdom (R. J. Hay); Department of Health Metrics Sciences, School of Medicine, University of Washington, Seattle (S. I. Hay, Mokdad, Reiner, Sartorius, Vollset, Vos, Murray); Mizan-Tepi University, Teppi, Ethiopia (Henok); Department of Statistics and Econometrics, Bucharest University of Economic Studies, Bucharest, Romania (Herteliu, Ileanu, Pana); Department of Epidemiology, Adigrat University, Adigrat, Ethiopia (Hidru); Center of Excellence in Behavioral Medicine, Nguyen Tat Thanh University, Ho Chi Minh City, Vietnam (Hoang, Vu); The University of Texas at Austin, Austin (Hole); Transdisciplinary Centre for Qualitative Methods, Manipal University, Manipal, India (Hoogar); Department of Pulmonology, Yokohama City University, Kanazawa-ku, Yokohama, Japan (Horita); National Human Genome Research Institute, National Institutes of Health, Bethesda, Maryland (Horita); Department of Epidemiology and Population Health, Albert Einstein College of Medicine, Bronx, NY (Hosgood); Department of Epidemiology and Biostatistics, Tehran University of Medical Sciences, Tehran, Iran (Hosseini, Mansournia, Yaseri); Department of Computer Engineering, Science and Research Branch, Islamic Azad University, Tehran, Iran (Hosseinzadeh); Department of Computer Science, University of Human Development, Sulaimaniyah, Iraq (Hosseinzadeh); Department of General Surgery, Carol Davila University of Medicine and Pharmacy, Bucharest, Romania (M. Hostiuc): Department of Internal Medicine, Bucharest Emergency Hospital, Bucharest, Romania (M. Hostiuc); Faculty of Dentistry, Department of Legal Medicine and Bioethics, Carol Davila University of Medicine and Pharmacy, Bucharest, Romania (S. Hostiuc); Department of Clinical Legal Medicine, National Institute of Legal Medicine Mina Minovici, Bucharest, Romania (S. Hostiuc); Division of Information and Computing Technology, College of Science and Engineering, Hamad Bin Khalifa University, Doha, Qatar (Househ); Qatar Foundation, Doha, Qatar (Househ); Department of Medical Laboratory Science, Arba Minch University, Arba Minch, Ethiopia (Hussen); Center for Health Outcomes \& Evaluation, Bucharest, Romania (Ileanu, Pana); Department of Epidemiology,
Faculty of Medical Sciences, University of Kragujevac, Kragujevac, Serbia (llic); Department of Epidemiology and Biostatistics, National Institute for Health Development, Tallinn, Estonia (Innos): Research Institute for Endocrine Sciences, Shahid Beheshti University of Medical Sciences, Tehran, Iran (Irvani); Department of Surgery, Usmanu Danfodiyo University Teaching Hospital, Sokoto, Nigeria (Iseh); Institute for Physical Activity and Nutrition, Deakin University, Burwood, Victoria, Australia (Islam); Sydney Medical School, University of Sydney, Sydney, New South Wales, Australia (Islam); Surveillance and Health Services Research, American Cancer Society, Atlanta, Georgia (Islami); Department of Psychosis, Babol Noshirvani University of Technology, Babol, Iran (Jafari Balalami); ,Department of Immunology, School of Medicine, Isfahan University of Medical Sciences, Isfahan, Iran (Jafarinia); Health Education and Health Promotion Department, Tabriz University of Medical Sciences, Tabriz, Iran (Jahangiry); Faculty of Medicine, Babol University of Medical Sciences, Babol, Iran (Jahani); School of Public Health, Shahid Beheshti University of Medical Sciences, Tehran, Iran (Jahanmehr); Safety Promotion and Injury Prevention Research Center, Shahid Beheshti University of Medical Sciences, Tehran, Iran (Jahanmehr); Medical Sciences Department, University of Kragujevac, Kragujevac, Serbia (Jakovljevic); Newcastle University, Tyne, United Kingdom (Javanbakht); Department of Surgery, Virginia Commonwealth University, Richmond (Jayaraman); Department of Public Health, Yonsei University, Seoul, South Korea (Jee); Harvard Medical School, Harvard University, Boston, Massachusetts (Jee); Faculty of Nursing \& Midwifery, Hamadan University of Medical Sciences, Hamadan, Iran (Jenabi); Department of Community Medicine, Banaras Hindu University, Varanasi, India (Jha); Department of Ophthalmology, Heidelberg University, Mannheim, Germany (Jonas); Beijing Institute of Ophthalmology, Beijing Tongren Hospital, Beijing, China (Jonas); School of Public Health and Community Medicine, University of New South Wales, Sydney, New South Wales, Australia (Jonnagaddala, Sitas); NSW Health, Sydney, New South Wales, Australia (Jonnagaddala); Health Services Management Training Centre, Semmelweis University, Budapest, Hungary (Joo); School of Health Sciences, Savitribai Phule Pune University, Pune, India (Jungari); Institute of Family Medicine and Public Health, University of Tartu, Tartu, Estonia (Jürisson); Minimally Invasive Surgery Research Center, Iran University of Medical Sciences, Tehran, Iran (Kabir); Department of Biology, Morgan State University, Baltimore, Maryland (Kamangar); Institute for Epidemiology and Social Medicine, University of Münster, Münster, Germany (Karch); Immunogenetics Research Center, Mazandaran University of Medical Sciences, Sari, Iran (Karimi); Department of Neurology, Mazandaran University of Medical Sciences, Sari, Iran (Karimi); Cellular and Molecular Biology Research Center, Babol University of Medical Sciences, Babol, Iran (Karimian); Drug Applied Research Center, Tabriz University of Medical Sciences, Tabriz, Iran (Karimian, Rahimi, Yousefi); Hematology-Oncology and Stem Cell Transplantation Research Center, Tehran University of Medical Sciences, Tehran, Iran (Kasaeian); Hematologic Malignancies Research Center, Tehran University of Medical Sciences, Tehran, Iran
(Kasaeian); Department of Pharmacology and Clinical Pharmacy, Addis Ababa University, Dessie, Ethiopia (B. Kassa); Department of Public Health, Amhara Public Health Institute, Bahir Dar, Ethiopia (Kassaw); School of Health Care Administration, Oklahoma State University, Tulsa (Kaul); Health Care Delivery Sciences, University of Tulsa, Tulsa, Oklahoma (Kaul); ODeL Campus, University of Nairobi, Nairobi, Kenya (Keiyoro); Department of Dermatology, Wolaita Sodo University, Wolaita Sodo, Ethiopia (Kelbore); Department of Public Health, Madda Walabu University, Goba, Ethiopia (Kerbo); School of Public Health, Wolaita Sodo University, Wolaita Sodo, Ethiopia (Kerbo); Department of Public Health and Community Medicine, Jordan University of Science and Technology, Ramtha, Jordan (Khader); Epidemiology and Biostatistics Department, Health Services Academy, Islamabad, Pakistan (E. A. Khan); Department of Medical Microbiology \& Immunology, United Arab Emirates University, Al Ain, United Arab Emirates (G. Khan); Division of Health Policy and Management, Seoul National University, Seoul, South Korea (Khang); Faculty of Health and Wellbeing, Sheffield Hallam University, Sheffield, United Kingdom (Khatab); College of Arts and Sciences, Ohio University, Zanesville (Khatab); Internal Medicine and Gastroenterology Department, National Hepatology and Tropical Research Institute, Cairo, Egypt (Khater); Academy of Medical Sciences, Tehran, Iran (Khayamzadeh); Department of Public Health, Mazandaran University of Medical Sciences, Sari, Iran (Khazaee-Pool); Health Sciences Research Center, Mazandaran University of Medical Sciences, Sari, Iran (Khazaee-Pool, Moosazadeh); Department of Epidemiology, Hamadan University of Medical Sciences, Hamadan, Iran (Khazaei); Department of Public Health, Imam Muhammad Ibn Saud Islamic University, Riyadh, Saudi Arabia (Khoja); Department of Health Policy and Management, Johns Hopkins University, Baltimore, Maryland (Khoja); Student Research Committee, Baqiyatallah University of Medical Sciences, Tehran, Iran (Khosravi); International Otorhinolaryngology Research Association, Tehran, Iran (Khosravi); Department of Nutrition and Health Science, Ball State University, Muncie, Indiana (Khubchandani); Department of Public Health, Kermanshah University of Medical Sciences, Kermanshah, Iran (Kianipour); Department of Health Sciences, Northeastern University, Boston, Massachusetts (D. Kim); School of Medicine, Xiamen University Malaysia, Sepang, Malaysia (Y. J. Kim); Department of Health Management and Health Economics, Kristiania University College, Oslo, Norway (A. Kisa); Department of Health Services Policy \& Management, University of South Carolina, Columbia (A. Kisa); Department of Nursing and Health Promotion, Oslo Metropolitan University, Oslo, Norway (S. Kisa); Department of Health Economics and Social Security, Jagiellonian University Medical College, Krakow, Poland (Kissimova-Skarbek); Neurophysiology Research Center, Hamadan University of Medical Sciences, Hamadan, Iran (Komaki); Brain Engineering Research Center, Institute for Research in Fundamental Sciences, Tehran, Iran (Komaki); Networking Center for Mental Health Network, San Juan de Dios Sanitary Park, Sant Boi de Llobregat, Spain (Koyanagi); Catalan Institution for Research and Advanced Studies, Barcelona, Spain (Koyanagi); Department of Public Health, Yüksek 
Ihtisas University, Ankara, Turkey (Bicer); Department of Public Health, Hacettepe University, Ankara, Turkey (Bicer); Department of Psychology and Health Promotion, University of KwaZulu-Natal, Durban, South Africa (Kugbey); Department of Medicine Brigham and Women's Hospital, Harvard University, Boston,

Massachusetts (Kumar); Department of Nursing, St. John of God Hospital, Duayaw Nkwanta, Ghana (Kuupiel); Clinical Medicine and Community Health, A.C.S. Medical College and Hospital, Milan, Italy (La Vecchia); Department of Internal Medicine, Postgraduate Institute of Medical Education and Research, Chandigarh, India (Lad); Department of Epidemiology and Biostatistics, University of Gondar, Gondar, Ethiopia (Lakew); Department of Community and Family Medicine, Academy of Medical Science, Baghdad, Iraq (Lami); Division of Cancer Epidemiology and Genetics, National Cance Institute, Rockville, Maryland (Lan); Department of Otorhinolaryngology-Head and Neck Surgery, Father Muller Medical College, Mangalore, India (Lasrado); Institute of Clinical Physiology, Italian National Research Council, Pisa, Italy (Lauriola); Barcelona Institute for Global Health, Barcelona, Spain (Lazarus); Asbestos Diseases Research Institute, University of Sydney, Sydney, New South Wales, Australia (Leigh, Takahashi); Department of Public Health, Debre Markos University, Debre Markos, Ethiopia (Leshargie, Wonde); Department of Medical Statistics and Epidemiology, Sun Yat-sen University, Guangzhou, China (Liao); Alliance for Improving Health Outcomes, Inc, Quezon City, Philippines (Liao); Department of Dentistry, Radboud University, Nijmegen, Netherlands (Listl); Section for Translational Health Economics, Heidelberg University Hospital, Heidelberg, Germany (Listl); University of Melbourne, Melbourne, Queensland, Australia (Lopez) Department of General Surgery, Aintree University Hospital National Health Service Foundation Trust, Liverpool, England, United Kingdom (Lunevicius); Department of Surgery, University of Liverpool, Liverpool, England, United Kingdom (Lunevicius) Department of Pathology, College of Medicine, Imam Abdulrahman Bin Faisal University, Dammam, Saudi Arabia (Madadin); Proteomics and Metabolomics Unit, Suez Canal University, Cairo, Egypt (Magdeldin); Department of Physiology, Suez Canal University, Ismailia, Egypt (Magdeldin); Department of Cardiology, Damietta University, Damietta, Egypt (El Razek); Department of Primary Care and Public Health, Imperial College London, London, England, United Kingdom (Majeed, S. Rawaf); Department of Environmental Health, Tehran University of Medical Sciences, Tehran, Iran (Maleki); Environmental Health Research Center, Research Institute for Health Development, Kurdistan University of Medical Sciences, Sanandaj, Iran (Maleki); Digestive Diseases Research Institute, Tehran University of Medical Sciences, Tehran, Iran (Malekzadeh, Merat, Pourshams, Roshandel, Salimzadeh, Sepanlou); Non-communicable Diseases Research Center, Shiraz University of Medical Sciences, Shiraz, Iran (Malekzadeh, Sepanlou); Department of Plastic Surgery, Iran University of Medical Sciences, Tehran, Iran (A. Manafi); Department of Ophthalmology, Iran University of Medical Sciences, Tehran, Iran (N. Manafi); Department of Ophthalmology, University of Manitoba, Winnipeg, Manitoba, Canada (N. Manafi); Department of Health Education and Promotion, Iran University of Medical Sciences,
Tehran, Iran (Mansourian); School of Medicine and Surgery, University of Milan-Bicocca, Monza, Italy (Mantovani); School of Nursing and Midwifery, Guilan University of Medical Sciences, Rasht, Iran (Maroufizadeh); Department of Epidemiology, Airlangga University, Surabaya, Indonesia (Martini) Indonesian Public Health Association, Surabaya Indonesia (Martini); School of Public Health, University of Botswana, Gaborone, Botswana (Maswabi); Department of Epidemiology and Public Health, University College London, London, England, United Kingdom (Mathur); Department of Ophthalmology, Hywel Dda University Health Board, Carmarthen, Wales, United Kingdom (McAlinden); Department of Health Services Research and Policy, London School of Hygiene \& Tropical Medicine, London, England, United Kingdom (McKee); Department of Nursing, Debre Markos University, Debre Markos, Ethiopia (Meheretu, Wagnew); School of Public Health, Bahir Dar University, Bahir Dar, Ethiopia (Meheretu); Department of Preventive Oncology, National Institute of Cancer Prevention and Research, Noida, India (Mehrotra); Department of Internal Medicine, SevenHills Hospital, Mumbai, India (Mehta); Institute for Agricultural and Nutritional Sciences, Martin Luther University Halle-Wittenberg, Halle, Germany (Meier): Innovation Office, Competence Cluster for Nutrition and Cardiovascular Health, Halle, Germany (Meier); Adelaide Medical School, University of Adelaide, Adelaide, South Australia, Australia (Melaku); Department of Public Health and Epidemiology, Arba Minch University, Arba Minch, Ethiopia (G. G. Meles); Mekelle University, Mekelle, Ethiopia (H. G. Meles, Tassew, F. H. Tesfay); Department of Medical Laboratory Science, Bahir Dar University, Bahir Dar, Ethiopia (Melese); Department of Public Health, University of West Florida, Pensacola, Florida (Memiah); Peru Country Office, United Nations Population Fund, Lima, Peru (Mendoza); Forensic Medicine Division, Department of Pathology, College of Medicine, Imam Abdulrahman Bin Faisal University, Dammam, Saudi Arabia (Menezes); Breast Surgery Unit, Helsinki University Hospital, Helsinki, Finland (Meretoja); University of Helsinki, Helsinki, Finland (Meretoja); Clinical Microbiology and Parasitology Unit, Dr. Zora Profozic Polyclinic, Zagreb, Croatia (Mestrovic); University Centre Varazdin, University North, Varazdin, Croatia (Mestrovic); Center for Innovation in Medical Education, Pomeranian Medical University, Szczecin, Poland (B.

Miazgowski); Pomeranian Medical University, Szczecin, Poland (B. Miazgowski); Department of Hypertension, Pomeranian Medical University, Szczecin, Poland (T. Miazgowski); Department of Epidemiology, Bahir Dar University, Bahir Dar, Ethiopia (Mihretie); Pacific Institute for Research and Evaluation, Calverton, Maryland (Miller); School of Public Health, Curtin University, Perth, Western Australia, Australia (Miller); Department of Health Research Methods, Evidence, and Impact, McMaster University, Hamilton, Ontario, Canada (Mills); Golestan University of Medical Sciences, Golestan, Iran (Mir); Research Center for Biochemistry and Nutrition in Metabolic Diseases, Kashan University of Medical Sciences, Kashan, Iran (H. Mirzaei, Tamtaji); Department of Medical Immunology, Tehran University of Medical Sciences, Tehran, Iran (H. R. Mirzaei); Department of Oral Medicine, University of Washington, Seattle, (Mishra); Institute of Public Health, Heidelberg
University, Heidelberg, Germany (Moazen, S. Mohammed); Institute of Addiction Research, Frankfurt University of Applied Sciences, Frankfurt, Germany (Moazen); Department of Biology, Salahaddin University, Erbil, Iraq (D. K. Mohammad, K. A. Mohammad); Department of Medicine, Huddinge, Karolinska Institutet, Stockholm, Sweden (D. K. Mohammad); ISHIK University, Erbil, Iraq (K. A. Mohammad); Department of Internal Medicine, King Saud University, Riyadh, Saudi Arabia (Y. Mohammad); Department of Information Technology, University of Human Development, Sulaymaniyah, Iraq (Darwesh); Department of Neurology, Kermanshah University of Medical Sciences, Kermanshah, Iran (H. Mohammadi); Department of Physiology and Pharmacology, Mazandaran University of Medical Sciences, Sari, Iran (M. Mohammadi); Department of Epidemiology and Biostatistics, Bushehr University of Medical Sciences, Bushehr, Iran (Mohammadian); Department of Epidemiology and Biostatistics, Shahrekord University of Medical Sciences, Shahrekord, Iran (Mohammadian-Hafshejani); Department of Clinical Biochemistry, Tarbiat Modares University, Tehran, Iran (Mohammadoo-Khorasani, Mosapour); Department of Nursing, Shahroud University of Medical Sciences, Shahroud, Iran (Mohammadpourhodki); Department of Public Health, Samara University, Samera, Ethiopia (J. A Mohammed); Health Systems and Policy Research Unit, Ahmadu Bello University, Zaria, Nigeria (S. Mohammed); Iran National Institute of Health Research, Tehran University of Medical Sciences, Tehran, Iran (Mohebi); Clinical Epidemiology and Public Health Research Unit, Burlo Garofolo Institute for Maternal and Child Health, Trieste, Italy (Monasta, Ronfani, Traini); Department of Molecular Medicine, Birjand University of Medical Sciences, Birjand, Iran (Moossavi); Social Determinants of Health Research Center, Kurdistan University of Medical Sciences, Sanandaj, Iran (Moradi, Moradpour); Department of Epidemiology and Biostatistics, Kurdistan University of Medical Sciences, Sanandaj, Iran (Moradi); Department of Economics and Management Sciences for Health, Tehran University of Medical Sciences, Tehran, Iran (Moradi-Joo); International Laboratory for Air Quality and Health, Queensland University of Technology, Brisbane, Queensland, Australia (Morawska); Hospital de Santo António, Hospital Center of Porto, Porto, Portugal

(Morgado-da-Costa); Department of Social Medicine, National Center for Child Health and Development, Setagaya, Japan (Morisaki); Department of Surgery, University of Washington, Seattle, (Morrison); Department of Health Management and Economics, Tehran University of Medical Sciences, Tehran, Iran (Mousavi); Department of Pharmacology and Clinical Pharmacy, Addis Ababa University, Addis Ababa, Ethiopia (Muhammed, Wondafrash); Department of Obstetrics and Gynecology, University of Jos, Jos, Nigeria (Musa); Center for Global Health, Northwestern University, Chicago, Illinois (Musa); Department of Obstetrics and Gynecology, Ain Shams University, Cairo, Egypt (Nabhan); Knowledge Translation and Utilization, Egyptian Center for Evidence Based Medicine, Cairo, Egypt (Nabhan); School of Paramedical Sciences, Kermanshah University of Medical Sciences, Kermanshah, Iran (Naderi); Department of Research and Analytics, Initiative for Financing 
Health and Human Development, Chennai, India (Nagarajan); Department of Research and Analytics, Bioinsilico Technologies, Chennai, India (Nagarajan); Institute of Epidemiology and Medical Biometry, Ulm University, Ulm, Germany (Nagel); Cancer Institute, Tehran University of Medical Sciences, Tehran, Iran (Nahvijou, Shirkoohi, Zendehdel); O'Neal Comprehensive Cancer Center, University of Alabama at Birmingham (Naik); Department of Epidemiology and Biostatistics, Kermanshah University of Medical Sciences, Kermanshah, Iran (Najafi); Department of Dermatology, San Bortolo Hospital, Vicenza, Italy (Naldi); GISED Study Center, Bergamo, Italy (Naldi); Department of Preventive Medicine and Public Health, Chungnam National University School of Medicine, Daejeon, South Korea (Nam); Daejeon Regional Cancer Center, Chungnam National University Hospital, Daejeon, South Korea (Nam); Department of Public Health, School of Public Health, Jiroft University of Medical Sciences, Jiroft Iran (Nasiri); Department of Pediatrics, Arak University of Medical Sciences, Arak, Iran (Nazari) Ministry of Health and Medical Education, Tehran, Iran (Nazari); Faculty of Health Sciences, University of Tampere, Tampere, Finland (Neupane); Public Health Sciences Division, Fred Hutchinson Cancer Research Center, Seattle, Washington (Newcomb); Department of Epidemiology, University of Washington, Seattle, (Newcomb, Oren); Department of Histopathology, University of Maiduguri Teaching Hospital, Maiduguri, Nigeria (Nggada); Department of Human Pathology, University of Maiduguri, Maiduguri, Nigeria (Nggada); Department of Biological Sciences University of Embu, Embu, Kenya (Ngunjiri); Institute for Global Health Innovations, Duy Tan University, Hanoi, Vietnam (Nguyen); Tabriz Health Services Management Research Center, Tabriz University of Medical Sciences, Tabriz, Iran (Nikniaz); Department of Public Health Sciences, State University of Semarang, Semarang, Indonesia (Ningrum); Graduate Institute of Biomedical Informatics, Taipei Medical University, Taipei City, Taiwan (Ningrum); Cochrane South Africa, South African Medical Research Council, Cape Town, South Africa (Nnaji); School of Public Health and Family Medicine, University of Cape Town, Cape Town, South Africa (Nnaji); Department of Community and Family Medicine, Iran University of Medical Sciences, Tehran, Iran (Nojomi); Department of Health Economics, Tabriz University of Medical Sciences, Tabriz, Iran (Nosratnejad); Mazandaran University of Medical Sciences, Sari, Iran (Shiadeh); Department of Anesthesia, Wolaita Sodo University, Sodo, Ethiopia (Obsa); Centre of Cardiovascular Research and Education in Therapeutics, Monash University, Melbourne, Victoria, Australia (Ofori-Asenso); Independent consultant, Accra, Ghana (Ofori-Asenso); Translational Health Research Institute, Western Sydney University, Penrith, New South Wales, Australia (Ogbo); Department of Preventive Medicine, Kyung Hee University, Dongdaemun-gu, South Korea (Oh); Department of Psychiatry and Behavioural Neurosciences, McMaster University, Hamilton, Ontario, Canada (A. T. Olagunju); Department of Psychiatry, University of Lagos, Lagos, Nigeria (A. T. Olagunju); Department of Pathology and Molecular Medicine, McMaster University, Hamilton, Ontario, Canada (T. O. Olagunju); Department of Health Promotion and Education, Faculty of Public Health, College of
Medicine, University of Ibadan, Ibadan, Nigeria (Oluwasanu); Department of Anatomic Pathology, Ekiti State University, Ado- Ekiti, Nigeria (Omonisi) Department of Anatomic Pathology, Ekiti State University Teaching Hospital, Ado-Ekiti, Nigeria (Omonisi); Department of Pharmacology and Therapeutics, University of Nigeria, Nsukka, Enugu, Nigeria (Onwujekwe); Department of Community Health, Christian Medical College, Vellore, India (Oommen); Graduate School of Public Health, San Diego State University, San Diego, California (Oren); Department of Global Health Nursing, St. Luke's International University, Chuo-ku, Japan (Ota); Analytical Center, Moscow Institute of Physics and Technology, Dolgoprudny, Russia (Otstavnov); Institute for Advanced Medical Research and Training, University of Ibadan, Ibadan, Nigeria (Owolabi); Department of TB \& Respiratory Medicine, Jagadguru Sri Shivarathreeswara University, Mysore, India (P A); Department of Forensic Medicine and Toxicology, Manipal University, Mangaluru, India (Padubidri); Department of Medicine, Ottawa Hospital Research Institute, Ottawa, Ontario, Canada (Pakhale); Department of Public Health, Qazvin University of Medical Sciences, Qazvin, Iran (Pakpour); Department of Nursing, Jönköping University, Jönköping, Sweden (Pakpour); Department of Medical Humanities and Social Medicine, Kosin University, Busan, South Korea (Park); Environmental Health Research Center, Research Institute for Health Development, Kurdistan University of Medical Sciences, Sanandaj, Iran (Pashaei); Department of Medicine, Maimonides Medical Center, Brooklyn, New York (Patel); Krishna Institute of Medical Sciences, Deemed University, Karad, India (Patil); University of Cartagena, Cartagena, Colombia (Pereira); Reference Center for Epidemiology and Cancer Prevention, CPO Piedmont, Torino, Italy (Piccinelli); Basic Medical Sciences Department, Durban University of Technology, Durban, South Africa (Pillay); Departments of Parasitology and Entomology, Faculty of Medical Sciences, Tarbiat Modares University, Tehran, Iran (Pirestani); Uro-Oncology Research Center, Tehran University of Medical Sciences, Tehran, Iran (Pishgar); University Medica Center Groningen, University of Groningen, Groningen, Netherlands (Postma); Faculty of Economics and Business, University of Groningen, Groningen, Netherlands (Postma); Department of Public Health, Maragheh University of Medical Sciences, Maragheh, Iran (Pourjafar); Department of Nutrition and Food Sciences, Maragheh University of Medical Sciences, Maragheh, Iran (Pourjafar); Department of Nephrology, Sanjay Gandhi Postgraduate Institute of Medical Sciences, Lucknow, India (Prakash, Prasad);

Non-communicable Diseases Research Center, Alborz University of Medical Sciences, Karaj, Iran (Qorbani); Department of Biomedical Engineering, Amirkabir University of Technology, Tehran, Iran (M. Rabiee); Divisions of Chemistry and Diseases, Advanced Technologies Research Group, Tehran, Iran (N. Rabiee); A.T. Still University, Mesa, Arizona (Radfar); Department of Immunology, Mazandaran University of Medical Sciences, Sari, Iran (Rafiei) Molecular and Cell Biology Research Center, Mazandaran University of Medical Sciences, Sari, Iran (Rafiei); Thalassemia and Hemoglobinopathy Research Center, Ahvaz Jundishapur University of Medical Sciences, Ahvaz, Iran (Rahim); Austin Health Clinical School of Nursing, La Trobe
University, Heidelberg, Victoria, Australia (Rahman); National Centre for Farmer Health, Deakin University, Waurn Ponds, Victoria, Australia (Rahman); Department of Health Education and Promotion, Kermanshah University of Medical Sciences, Kermanshah, Iran (Rajati); Department of Public Health, Contech School of Public Health, Lahore, Pakistan (Rana); Public Health Department, University of Health Sciences, Lahore, Pakistan (Rana); Department of Radiation Oncology, All India Institute of Medical Sciences, New Delhi, India (Rath); WHO Collaborating Centre for Public Health Education and Training, Imperial College London, London, England, United Kingdom (D. L. Rawaf); University College London Hospitals, London, England, United Kingdom (D. L. Rawaf); Academic Public Health, Public Health England, London, England, United Kingdom (S. Rawaf); School of Social Sciences and Psychology, Western Sydney University, Penrith, New South Wales, Australia (Renzaho); Research Center for Immunodeficiencies, Children's Medical Center, Tehran University of Medical Sciences, Tehran, Iran (Rezaei); Network of Immunity in Infection, Malignancy, and Autoimmunity, Universal Scientific Education and Research Network, Tehran, Iran (Rezaei); Health Management and Economics Research Center, Iran University of Medical Sciences, Tehran, Iran (Rezapour, Moghadam); Epidemiology Research Unit, Institute of Public Health, University of Porto, Porto, Portugal (A. I. Ribeiro); Department of Applied Chemistry, Faculty of Pharmacy, University of Porto, Porto, Portugal (D. Ribeiro); Department of Public Health, Wollega University, Nekemte, Ethiopia (Roro); Golestan Research Center of Gastroenterology and Hepatology, Golestan University of Medical Sciences, Gorgan, Iran (Roshandel); Infectious Diseases and Tropical Medicine Research Center, Babol University of Medical Sciences, Babol, Iran (Rostami, Sabbagh); Clinical Operations, Doctor Evidence, Santa Monica, California (Saad); Department of Epidemiology, Shahid Beheshti University of Medical Sciences, Tehran, Iran (Sabour); Medical Department, University of Sharjah, Sharjah, United Arab Emirates (Saddik); Managerial Epidemiology Research Center, Maragheh University of Medical Sciences, Maragheh, Iran (Safiri); Biotechnology Research Center, Mashhad University of Medical Sciences, Mashhad, Iran (Sahebkar); Neurogenic Inflammation Research Center, Mashhad University of Medical Sciences, Mashhad, Iran (Sahebkar); A.C.S. Medical College and Hospital, Tehran, Iran (Salahshoor); Taleghani Hospital, Kermanshah University of Medical Sciences, Kermanshah, Iran (Salehi); Department of Urology, Cairo University, Cairo, Egypt (H. Salem); Public Health and Community Medicine Department, Cairo University, Giza, Egypt (M. R. Salem); Center for Health Policy and Center for Primary Care and Outcomes Research, Stanford University, Stanford, California (Salomon); Department of Entomology, Ain Shams University, Cairo, Egypt (Samy); Department of Surgery, Marshall University, Huntington, West Virginia (Sanabria); Departments of Nutrition and Preventive Medicine, Case Western Reserve University, Cleveland, Ohio (Sanabria); School of Public Health and Health Management, University of Belgrade, Belgrade, Serbia (Santric Milicevic); Faculty of Infectious and Tropical Diseases, London School of Hygiene \& Tropical Medicine, London, England, United Kingdom (Sartorius); Colorectal 
Research Center, Iran University of Medical Sciences, Tehran, Iran (Sarveazad); Surgery Department, Hamad Medical Corporation, Doha, Qatar (Sathian); Faculty of Health \& Social Sciences Bournemouth University, Bournemouth, England, United Kingdom (Sathian); UGC Centre of Advanced Study in Psychology, Utkal University, Bhubaneswar, India (Satpathy); Udyam-Global Association for Sustainable Development, Bhubaneswar, India (Satpathy); GSK Biologicals, Wavre, Belgium (Savic); Department of Public Health Sciences, University of North Carolina at Charlotte (Sawhney); Education Development Center, Ahvaz Jundishapur University of Medical Sciences, Ahvaz, Iran (Sayyah); School of Health Sciences, Federal University of Santa Catarina, Ararangua, Brazil (Schneider); Department of Medical Statistics, Epidemiology and Medical Informatics, University of Zagreb, Zagreb, Croatia (Sekerija); Division of Epidemiology and Prevention of Chronic Noncommunicable Diseases, Croatian Institute of Public Health, Zagreb, Croatia (Sekerija); Gastrointestinal and Liver Diseases Research Center, Guilan University of Medical Sciences Rasht, Iran (Sepehrimanesh); Center of Expertise in Microbiology, Tehran University of Medical Sciences, Tehran, Iran (Seyedmousavi); Invasive Fungi Research Center, Mazandaran University of Medical Sciences, Sari, Iran (Seyedmousavi); Department of Health Promotion and Education, Alborz University of Medical Sciences, Karaj, Iran (Shaahmadi); Department of Health Policy, Iran University of Medical Sciences, Tehran, Iran (Shabaninejad); Department of Epidemiology, Shahid Beheshti University of Medical Sciences, Tehran, Iran (Shahbaz); Independent consultant, Karachi, Pakistan (Shaikh); Department of Medical Laboratory Sciences, Mazandaran University of Medical Sciences, Sari, Iran (Shamshirian); Chronic Diseases (Home Care) Research Center, Hamadan University of Medical Sciences, Hamadan, Iran (Shamsizadeh); Department of Molecular Hepatology, Middle East Liver Disease Center, Tehran, Iran (H. Sharafi); Razi Herbal Medicines Research Center, Lorestan University of Medical Sciences, Khorramabad, Iran (Z. Sharafi); Department of Basic Sciences, Islamic Azad University, Sari, Iran (Sharif); Department of Laboratory Sciences, Islamic Azad University, Sari, Iran (Sharif); Department of Ophthalmology, Kerman University of Medical Sciences, Kerman, Iran (A. Sharifi); HIV/STI Surveillance Research Center, Institute for Futures Studies in Health, Kerman University of Medical Sciences, Kerman, Iran (H. Sharifi); University School of Management and Entrepreneurship, Delhi Technological University, New Delhi, India (Sharma); Usher Institute of Population Health Sciences and Informatics, University of Edinburgh, Edinburgh, Scotland, United Kingdom (Sheikh); Division of General Internal Medicine and Primary Care, Harvard University, Boston, Massachusetts (Sheikh); Cancer Biology Research Center, Tehran University of Medical Sciences, Tehran, Iran (Shirkoohi); Symbiosis Institute of Health Sciences, Symbiosis International University, Pune, Maharashtra, India (Shukla); School of Public Health and Preventive Medicine, Monash University, Melbourne, Victoria, Australia (Si); Imam Al Cardiovascular Research Center, Kermanshah University of Medical Sciences, Kermanshah, Iran (Siabani); Faculty of Health, University of Technology Sydney, Sydney, New South Wales,
Australia (Siabani); School of Health Sciences, Federal University of Santa Catarina, Florianópolis, Brazil (Silva); University of Brasília, Brasília, Brazil (Silveira); Department of the Health Industrial Complex and Innovation in Health, Ministry of Health, Brasília, Brazil (Silveira); Menzies Institute for Medical Research, University of Tasmania, Hobart, Tasmania, Australia (A. Singh); Global Patient Outcome and Real World Evidence, Eli Lilly and Company, Indianapolis, Indiana (A. Singh) Department of Medicine, University of Alabama at Birmingham (J. A. Singh); Department of Epidemiology, University of Alabama at Birmingham (J. A. Singh); Medical Department, German Leprosy and TB Relief Association, Addis Ababa, Ethiopia (Sisay); Sydney School of Public Health, University of Sydney, Sydney, New South Wales, Australia (Sitas); Department of Internal Medicine and Specialties, University of Yaoundé I, Yaoundé, Cameroon (Sobngwi); Department of Endocrinology and Diabetes, Central Hospital of Yaoundé, Yaoundé, Cameroon (Sobngwi); Social Development and Health Promotion Research Center, Kermanshah University of Medical Sciences, Kermanshah, Iran (Soofi, Zangeneh); Hospital Universitario de la Princesa, Autonomous University of Madrid, Madrid, Spain (Soriano): Centro de Investigación en Red de Enfermedades Respiratorias, Institute of Health Carlos III, Madrid, Spain (Soriano); Department of Occupational Therapy, Athens University of Applied Sciences, Athens, Greece (Stathopoulou); Department of Community Medicine, Ahmadu Bello University, Zaria, Nigeria (Sufiyan); Department of Medicine, University of Valencia, Valencia, Spain (Tabarés-Seisdedos); Biomedical Research Networking Center for Mental Health Network, Carlos III Health Institute, Madrid, Spain (Tabarés-Seisdedos); Cancer Control Center, Osaka International Cancer Institute, Osaka, Japan (Tabuchi); University of Sydney, Sydney, New South Wales, Australia (Takahashi); Jordan University of Science and Technology, Ramtha, Jordan (Tarawneh); Department of Public Health, Kurdistan University of Medical Sciences, Sanandaj, Iran (Taymoori); Department of Community Medicine, Iran University of Medical Sciences, Tehran, Iran (Tehrani-Banihashemi); Department of Pediatrics, King Saud University, Riyadh, Saudi Arabia (M. Temsah); Department of Public Health, Adigrat University, Adigrat, Ethiopia (B. E. Tesfay); Southgate Institute for Health, Society and Equity, Flinders University, Adelaide, South Australia, Australia (F. H. Tesfay); Department of Public Health, Arba Minch University, Arba Minch, Ethiopia (Teshale); School of Public Health, University of Adelaide, Adelaide, South Australia, Australia (Tessema); Department of Public Health, University of Southern Denmark, Odense, Denmark (Thapa); Faculty of Health Sciences, Jagiellonian University Medical College, Krakow, Poland (Topor-Madry); The Agency for Health Technology Assessment and Tariff System, Warsaw, Poland (Topor-Madry); Department of Pathology and Legal Medicine, University of São Paulo, Ribeirão Preto, Brazil (Tovani-Palone); Department of Health Economics, Hanoi Medical University, Hanoi, Vietnam (B. X. Tran); Department of Molecular Medicine and Pathology, The University of Auckland, Auckland, New Zealand (K. B. Tran); Department of Clinical Hematology and Toxicology, Military Medical University, Hanoi, Vietnam (K. B. Tran); Gomal Center of Biochemistry and Biotechnology, Gomal
University, Dera Ismail Khan, Pakistan (Ullah) Division of Health Sciences, University of Warwick, Coventry, England, United Kingdom (Uthman); Department of General Surgery and Medical-Surgical Specialties, University of Catania, Catania, Italy (Vacante); Women's Reproductive Health Research Center, A.C.S. Medical College and Hospital, Tabriz, Iran (Vaezi); Alzahra Teaching Hospital, Tabriz, Iran (Vaezi); Department for International Development, Health Network of Cuba, Havana, Cuba (Varona Pérez); Centre of Research in Environmental Epidemiology, Barcelona Institute for Global Health, Barcelona, Spain (Varona Pérez); Psychosocial Injuries Research Center, Ilam University of Medical Sciences, Ilam, Iran (Veisani); Department of Neurology \& Stroke Unit, Sant'Anna Hospital, Como, Italy (Vidale); Occupational Health Unit, Sant'Orsola Malpighi Hospital, Bologna, Italy (Violante); Department of Health Care Administration and Economy, National Research University Higher School of Economics, Moscow, Russia (Vlassov); Department of Gastroenterology and Hepatology, Johns Hopkins University, Baltimore, Maryland (Vosoughi); Department of Pathology, Makerere University, Kampala, Uganda (Wabinga); Department of Public Health, Woldia University, Woldia, Ethiopia (Wachamo, Yimam); Foundation University Medical College, Foundation University, Rawalpindi, Pakistan (Waheed). Department of Psychology and Counselling, University of Melbourne, Melbourne, Victoria, Australia (Wijeratne); Department of Medicine, University of Melbourne, St Albans, Victoria, Australia (Wijeratne); Department of Pharmacology and Toxicology, Mekelle University, Mekelle, Ethiopia (Wondafrash); Department of Nursing, Wollo University, Dessie, Ethiopia (Wondmieneh); Department of Nursing, Addis Ababa University, Addis Ababa, Ethiopia (Wondmieneh); Department of Neonatal and Pediatric Health Nursing, Bahir Dar University, Bahirdar, Ethiopia (Workie); Department of Population Studies, International Institute for Population Sciences, Mumbai, India (Yadav); Foodborne and Waterborne Diseases Research Center, Shahid Beheshti University of Medical Sciences, Tehran, Iran (Yadegar); Department of Medical Physics, Ahvaz Jundishapur University of Medical Sciences, Ahvaz, Iran (Yadollahpour); Ophthalmic Research Center, Shahid Beheshti University of Medical Sciences, Tehran, Iran (Yaseri); Department of Health Management, Policy, and Economics, Kerman University of Medical Sciences, Kerman, Iran (Yazdi-Feyzabadi); Health Services Management Research Center, Institute for Futures Studies in Health, Kerman University of Medical Sciences, Kerman, Iran (Yazdi-Feyzabadi); Wolkite University, Wolkite, Ethiopia (Yeshaneh); School of Allied Health Sciences, Addis Ababa University, Addis Ababa, Ethiopia (Yisma); Department of Neuropsychopharmacology, National Center of Neurology and Psychiatry, Tokyo, Japan (Yonemoto); Health Economics \& Finance, Global Health, Jackson State University, Jackson, Mississippi (Younis); Department of Public Health, Tsinghua University, Beijing, China (Younis); Department of Clinical Biochemistry, Tabriz University of Medical Sciences, Tabriz, Iran (Yousefi); Physiology Research Center, Iran University of Medical Sciences, Tehran, Iran (Yousefifard); Department of Epidemiology and Biostatistics, Wuhan University, Wuhan, China (Yu); Global Health Institute, Wuhan University, Wuhan, 
China (Yu); Department of Electrical Engineering. Institute for Research in Fundamental Sciences, Tehran, Iran (Zabeh); Department of Electrical Engineering, Bioelectric Group, Sharif University of Technology, Tehran, Iran (Zabeh); Epidemiology and Cancer Registry Sector, Institute of Oncology. Ljubljana, Slovenia (Zadnik); Social Determinants of Health Research Center, Ardabil University of Medical Science, Ardabil, Iran (Moghadam, Zandian); Department of Epidemiology, University Hospital of Setif, Setif, Algeria (Zaidi); Student Research Committee, Babol University of Medical Sciences, Babol, Iran (Zamani); Department of Parasitology, Tarbiat Modares University, Tehran, Iran (L. Zaki); Department of Midwifery, Mekelle University, Mekelle, Ethiopia (Zenebe); Department of Epidemiology and Biostatistics, Bahir Dar University, Bahir Dar, Ethiopia (Zewale); Health Promotion Research Center, Iran University of Medical Sciences, Tehran, Iran (Ziapour).

Author Contributions: Dr Fitzmaurice had full access to all of the data in the study and takes responsibility for the integrity of the data and the accuracy of the data analysis. The authors had access to the data in the study and the final responsibility to submit for publication. Study concept and design: Fitzmaurice, Abbastabar, Abd-Allah, Abdelalim, Abdollahpour, Abualhasan, Alene, E. Amini, Anbari, Andrei, Ansariadi, Ataeinia, Ausloos, Babaee, Bagherzadeh, Bärnighausen, Belayneh, Bogale, Chanie, Chaturvedi, Constantin, Derakhshani, Deribe, Desta, Dinberu, Djalalinia, El Sayed, Endalew, Fentahun, Geta, Gezae, Ghashghaee, S. Hay, Herteliu, Hidru, Hosseinzadeh, Househ, James, Jee, Jenabi, Joo, Karimian, Khalilarjmandi, Khater, Kianipour, A. Kisa, S. Kisa, Lasrado, Leigh, Madadin, Maleki, N. Manafi, Manamo, Mansournia, Maroufizadeh, Mehrotra, Melaku, Menezes, Merat, Mills, Mir, Mohammad Darwesh, Mohammadoo-Khorasani, Mohammadpourhodki, A. Mohammed, Najafi, Nazari, Nggada, Omonisi, Postma, M. Rabiee, N. Rabiee, Rahim, Rana, Raoofi, Rath, D. Rawaf, Rezaei, Sabbagh, Salomon, Samy, Sanabria, Satpathy, Sayyah, Shahbaz, Shamshirian, Siabani, Sisay, Sitas, Tamtaji, Tarawneh, Tassew, B. Tesfay, Topor-Madry, Tovani-Palone, Varona Pérez, Wachamo, Yeshaneh, Zangeneh, Zewale, Murray. Acquisition, analysis, or interpretation of data: Fitzmaurice, Abate, Abbasi, Abdel-Rahman, Abdoli, Abdulle, Abebe, Abraha, Abu-Raddad, Adedeji, Advani, Afarideh, Afshari, Aghaali, Agius, Agrawal, Ahmadi, Ahmadian, Ahmadpour, Ahmed, Akbari, Akinyemiju, Al-Aly, AlAbdulKader, Alahdab, Alam, Alamene, Alemnew, Alinia, Alipour, Aljunid, Allah Bakeshei, Almadi, Almasi-Hashiani, Alsharif, Alsowaidi, Alvis-Guzman, S. Amini, Amoako, Anber, Anjomshoa, Ansari, Appiah, Arab-Zozani, Arabloo, Arefi, Aremu, Areri, Artaman, Asayesh, Asfaw, Ashagre, Assadi, Atalay, Ataro, Atique, Avila-Burgos, Avokpaho, Awasthi, Awoke, Ayala Quintanilla, Ayanore, Ayele, Bacha, Badawi, Bagherzadeh, Bagli, Balakrishnan, Balouchi, Bärnighausen, Battista, Masoud Behzadifar, Meysam Behzadifar, Bekele, Belay, Berfield, Berhane, Bernabé, Beuran, Bhakta, Bhattacharyya, Biadgo, Bijani, Bin Sayeed, Birungi, Bisignano, Bitew, Bjørge, Bleyer, Bogale, Bojia, Borzì, Bosetti, Bou-Orm, Brenner, Brewer, A. Briko, N. Briko, Bustamante-Teixeira, Butt, Carreras, Carrero, Carvalho, C. Castro, F. Castro, Catalá-López, Cerin, Chaiah, Chattu, Chauhan, Chehrazi, Chiang, Chichiabellu, Chido-Amajuoyi, Chimed-Ochir, Choi, Christopher, Chu, Costa,
Crocetti, Crowe, Curado, Dahlwai, Damiani, Darwish, Daryani, das Neves, Demeke, Demis, Demissie, Demoz, Denova-Gutiérrez, Deribe, Desai, Desalegn, Dey, Dharmaratne, Dhimal, Diaz, Doku, Drake, Dubey, Dubljanin, Duken, Ebrahimi, Effiong Eftekhari, El Razek, El Sayed, El Sayed Zaki, Elemineh, El-Jaafary, El-Khatib, Elkout, Ellenbogen, Elsharkawy, Emamian, Endries, Eshrati, Fadhil, Fallah Omrani, Faramarzi, Farhangi, Farioli, Farzadfar, Fernandes, Feyissa, Filip, Fischer, Fisher, Force, Foroutan, Freitas, Fukumoto, Futran, Gallus, Gankpe, Gayesa, Gebrehiwot, Gebremeskel, Gedefaw, Gelaw, Getachew, Ghafourifard, Ghajar, Gholamian, Gill, Ginindza, Girmay, Gizaw, Gomez, Gopalani, Gorini, Goulart, Grada, Guerra, Guimaraes, P. Gupta, R. Gupta, Hadkhale, Arvin Haj-Mirzaian, Arya Haj-Mirzaian, Hamadeh, Hamidi, Hanfore, Haro, Hasankhani, Hasanzadeh, Hassen, R. Hay, S. Hay, Henok, Henry, Hidru, Hoang, Hole, Hoogar, Horita, Hosgood, Hosseini, M. Hostiuc, S. Hostiuc, Hussen, Ileanu, Ilic, Innos, Irvani, Iseh, Islam, Islami, Jafari Balalami, Jafarinia, Jahangiry, Jahani, Jahanmehr, Jakovljevic, James, Javanbakht, Jayaraman, Jha, Jonas, Jonnagaddala, Jungari, Jürisson, Kabir, Kamangar, Karch, Karimi, Kasaeian, Kasahun, B. Kassa, T. Kassa, Kassaw, Kaul, Keiyoro, Kelbore, Kerbo, Khader, E. Khan, G. Khan, Khang, Khatab, Khater, Khayamzadeh, Khazaee-Pool, Khazaei, Khoja, Khosravi, Khubchandani, D. Kim, Y. Kim, A. Kisa, S. Kisa, Kissimova-Skarbek, Komaki, Koyanagi, Krohn, Kucuk Bicer, Kugbey, Kumar, Kuupiel, La Vecchia, Lad, Lake, Lakew, Lal, Lami, Lan, Lauriola, Lazarus, Leigh, Leshargie, Liao, Limenih, Listl, Lopez, Lopukhov, Lunevicius, Magdeldin, Majeed, Malekzadeh, A. Manafi, N. Manafi, Manamo, Mansourian, Mantovani, Martini, Mashamba-Thompson, Massenburg, Maswabi, Mathur, McAlinden, McKee, Meheretu, Mehta, Meier, G. Meles, H. Meles, Melese, Melku, Memiah, Mendoza, Merat, Meretoja, Mestrovic, B. Miazgowski, T. Miazgowski, Mihretie, Miller, Hamed Mirzaei, Hamid Reza Mirzaei, Mishra, Moazen, D. Mohammad, K. Mohammad, Y. Mohammad, Mohammadbeigi, H. Mohammadi, M. Mohammadi, Mohammadian, Mohammadian-Hafshejani, Mohammadoo-Khorasani, A. Mohammed, J. Mohammed, S. Mohammed, Mohebi, Mokdad, Monasta, Moodley, Moosazadeh, Moossavi, Moradi, Moradi-Joo, Moradi-Lakeh, Moradpour, Morawska, Morgado-da-Costa, Morisaki, Morrison, Mosapour, Mousavi, Muche, Muhammed, Musa, Nabhan, Naderi, Nagarajan, Nagel, Nahvijou, Naik, Naldi, Nam, Nasiri, Negoi, Neupane, Newcomb, Ngunjiri, Nguyen, Nikniaz, Ningrum, Nirayo, Nixon, Nnaji, Nojomi, Nosratnejad, Nourollahpour Shiadeh, Obsa, Ofori-Asenso, Ogbo, Oh, A. Olagunju, T. Olagunju, Oluwasanu, Onwujekwe, Oommen, Oren,

Ortega-Altamirano, Ota, Otstavnov, Owolabi, PA, Padubidri, Pakhale, Pakpour, Pana, Park, Parsian, Pashaei, Patel, Patil, Pennini, Pereira, Piccinelli, Pillay, Pirestani, Pishgar, Pourjafar, Pourmalek, Pourshams, Prakash, Prasad, Qorbani, M. Rabiee, N. Rabiee, Radfar, Rafiei, Rahimi, Rahman, Rajati, Raoofi, S. Rawaf, Reiner, Renzaho, Rezapour, A. Ribeiro, D. Ribeiro, Ronfani, Roro, Roshandel, Rostami, Saad, Sabbagh, Sabour, Saddik, Safiri, Sahebkar, Salehi, H. Salem, M. Salem, Salimzadeh, Samy, Santric Milicevic, Sartorius, Sarveazad, Sathian, Savic, Sawhney, Schneider, Schöttker, Sekerija, Sepanlou, Sepehrimanesh, Seyedmousavi, Shaahmadi, Shabaninejad, Shaikh, Shamsizadeh, H. Sharafi, Z. Sharafi, Sharif, A. Sharifi, H. Sharifi, Sharma, Sheikh, Shirkoohi, Shukla, Si, Siabani, Silva,
Silveira, A. Singh, J. Singh, Sisay, Sobngwi, Soofi, Soriano, Stathopoulou, Sufiyan, Tabarés-Seisdedos, Tabuchi, Takahashi, Taymoori,

Tehrani-Banihashemi, M. Temsah, O. Temsah, B. Tesfay, F. Tesfay, Teshale, Tessema, Thapa, Tlaye, Tovani-Palone, Traini, B. Tran, K. Tran, Tsadik, Ullah, Uthman, Vacante, Vaezi, Veisani, Vidale, Violante, Vlassov, Vollset, Vos, Vosoughi, Vu, Vujcic, Wabinga, Wachamo, Wagnew, Waheed, Weldegebreal, Weldesamuel, Wijeratne, Wondafrash, Wonde, Wondmieneh, Workie, Yadav, Yadegar, Yadollahpour, Yaseri, Yazdi-Feyzabadi, Yimam, Yimer, Yisma, Yonemoto, Younis, Yousefi, Yousefifard, Yu, Zabeh, Zadnik, Zahirian Moghadam, Zaidi, Zamani, Zandian, Zaki, Zendehdel, Zenebe, Zewale, Ziapour, Zodpey. Drafting of the manuscript: Fitzmaurice, Abu-Raddad, Alam, Aljunid, E. Amini, Anbari, Anber, Bagherzadeh, Bogale, Borzì, Chaturvedi, Deribe, Drake, El Razek, El Sayed, El Sayed Zaki, Fallah Omrani, Farhangi, Gelaw, Girmay, Arvin Haj-Mirzaian, Hasankhani, Househ, Jahanmehr, Jenabi, Khalilarjmandi, Khater, Khazaee-Pool, Komaki, Krohn, Kucuk Bicer, Massenburg, Maswabi, Mills, K. Mohammad, Mohammadpourhodki, Mokdad, Nagarajan, Omonisi, Pashaei, Pillay, M. Rabiee, N. Rabiee, Raoofi, Sabbagh, Salehi, Samy, Sanabria, Satpathy, Sayyah, Tassew, F. Tesfay, Uthman, Veisani, Wabinga, Wachamo, Yousefi, Zangeneh.

Critical revision of the manuscript for important intellectual content: Abate, Abbasi, Abbastabar, Abd-Allah, Abdel-Rahman, Abdelalim, Abdoli, Abdollahpour, Abdulle, Abebe, Abraha, Abualhasan, Adedeji, Advani, Afarideh, Afshari, Aghaali, Agius, Agrawal, Ahmadi, Ahmadian, Ahmadpour, Ahmed, Akbari, Akinyemiju, Al-Aly, AlAbdulKader, Alahdab, Alamene, Alemnew, Alene, Alinia, Alipour, Aljunid, Allah Bakeshei, Almadi, Almasi-Hashiani, Alsharif, Alsowaidi, Alvis-Guzman, S. Amini, Amoako, Anbari, Andrei, Anjomshoa, Ansari, Ansariadi, Appiah, Arab-Zozani, Arabloo, Arefi, Aremu, Areri, Artaman, Asayesh, Asfaw, Ashagre, Assadi, Ataeinia, Atalay, Ataro, Atique, Ausloos, Avila-Burgos, Avokpaho, Awasthi, Awoke, Ayala Quintanilla, Ayanore, Ayele, Babaee, Bacha, Badawi, Bagherzadeh, Bagli, Balakrishnan, Balouchi, Bärnighausen, Battista, Masoud Behzadifar, Meysam Behzadifar, Bekele, Belay, Belayneh, Berfield, Berhane, Bernabé, Beuran, Bhakta, Bhattacharyya, Biadgo, Bijani, Bin Sayeed, Birungi, Bisignano, Bitew, Bjørge, Bleyer, Bojia, Borzì, Bosetti, Bou-Orm, Brenner, Brewer, A. Briko, N. Briko, Bustamante-Teixeira, Butt, Carreras, Carrero, Carvalho, C. Castro, F. Castro, Catalá-López, Cerin, Chaiah, Chanie, Chattu, Chauhan, Chehrazi, Chiang, Chichiabellu, Chido-Amajuoyi, Chimed-Ochir, Choi, Christopher, Chu, Constantin, Costa, Crocetti, Crowe, Curado, Dahlwai, Damiani, Darwish, Daryani, das Neves, Demeke, Demis, Demissie, Demoz,

Denova-Gutiérrez, Derakhshani, Deribe, Desai, Desalegn, Desta, Dey, Dharmaratne, Dhimal, Diaz, Dinberu, Djalalinia, Doku, Drake, Dubey, Dubljanin, Duken, Ebrahimi, Effiong, Eftekhari, El Razek, El Sayed, El Sayed Zaki, Elemineh, El-Jaafary, El-Khatib, Elkout, Ellenbogen, Elsharkawy, Emamian, Endalew, Endries, Eshrati, Fadhil, Faramarzi, Farioli, Farzadfar, Fentahun, Fernandes, Feyissa, Filip, Fischer, Fisher, Force, Foroutan, Freitas, Fukumoto, Futran, Gallus, Gankpe, Gayesa, Gebrehiwot, Gebremeskel, Gedefaw, Geta, Getachew, Gezae, Ghafourifard, Ghajar, Ghashghaee, Gholamian, Gill, Ginindza, Gizaw, 
Gomez, Gopalani, Gorini, Goulart, Grada, Guerra, Guimaraes, P. Gupta, R. Gupta, Hadkhale, Arvin Haj-Mirzaian, Arya Haj-Mirzaian, Hamadeh, Hamidi, Hanfore, Haro, Hasankhani, Hasanzadeh, Hassen, R. Hay, S. Hay, Henok, Henry, Herteliu, Hidru, Hoang, Hole, Hoogar, Horita, Hosgood, Hosseini, Hosseinzadeh, M. Hostiuc, S. Hostiuc, Househ, Hussen, Ileanu, Ilic, Innos, Irvani, Iseh, Islam, Islami, Jafari Balalami, Jafarinia, Jahangiry, Jahani, Jakovljevic, James, Javanbakht, Jayaraman, Jee, Jenabi, Jha, Jonas, Jonnagaddala, Joo, Jungari, Jürisson, Kabir, Kamangar, Karch, Karimi, Karimian, Kasaeian, Kasahun, B. Kassa, T. Kassa, Kassaw, Kaul, Keiyoro, Kelbore, Kerbo, Khader, E. Khan, G. Khan, Khang, Khatab, Khater, Khayamzadeh, Khazaee-Pool, Khazaei, Khoja, Khosravi, Khubchandani, Kianipour, D. Kim, Y. Kim, A. Kisa, S. Kisa, Kissimova-Skarbek, Komaki, Koyanagi, Krohn, Kugbey, Kumar, Kuupiel, La Vecchia, Lad, Lake, Lakew, Lal, Lami, Lan, Lasrado, Lauriola, Lazarus, Leigh, Leshargie, Liao, Limenih, Listl, Lopez, Lopukhov, Lunevicius, Madadin, Magdeldin, Majeed, Maleki, Malekzadeh, A. Manafi, N. Manafi, Manamo, Mansourian, Mansournia, Mantovani, Maroufizadeh, Martini, Mashamba-Thompson, Massenburg, Maswabi, Mathur, McAlinden, McKee, Meheretu, Mehrotra, Mehta, Meier, Melaku, G. Meles, H. Meles, Melese, Melku, Memiah, Mendoza, Menezes, Merat, Meretoja, Mestrovic, B. Miazgowski, T. Miazgowski, Mihretie, Miller, Mills, Mir, Hamed Mirzaei, Hamid Reza Mirzaei, Mishra, Moazen, D. Mohammad, Y. Mohammad, Mohammad Darwesh, Mohammadbeigi, $\mathrm{H}$ Mohammadi, M. Mohammadi, Mohammadian, Mohammadian-Hafshejani,

Mohammadoo-Khorasani, Mohammadpourhodki, A. Mohammed, J. Mohammed, S. Mohammed, Mohebi, Mokdad, Monasta, Moodley, Moosazadeh, Moossavi, Moradi, Moradi-Joo, Moradi-Lakeh, Moradpour, Morawska, Morgado-da-Costa, Morisaki, Morrison, Mosapour, Mousavi, Muche, Muhammed, Musa, Nabhan, Naderi, Nagarajan, Nagel, Nahvijou, Naik, Najafi, Naldi, Nam, Nasiri, Nazari, Negoi, Neupane, Newcomb, Nggada, Ngunjiri, Nguyen, Nikniaz, Ningrum, Nirayo, Nixon, Nnaji, Nojomi, Nosratnejad, Nourollahpour Shiadeh, Obsa, Ofori-Asenso, Ogbo, Oh, A. Olagunju, T. Olagunju, Oluwasanu, Onwujekwe, Oommen, Oren, Ortega-Altamirano, Ota, Otstavnov, Owolabi, PA, Padubidri, Pakhale, Pakpour, Pana, Park, Parsian, Pashaei, Patel, Patil, Pennini, Pereira, Piccinelli, Pillay, Pirestani, Pishgar, Postma, Pourjafar, Pourmalek, Pourshams, Prakash, Prasad, Qorbani M. Rabiee, N. Rabiee, Radfar, Rafiei, Rahim, Rahimi, Rahman, Rajati, Rana, Raoofi, Rath, D. Rawaf, S. Rawaf, Reiner, Renzaho, Rezaei, Rezapour, A. Ribeiro, D. Ribeiro, Ronfani, Roro, Roshandel, Rostami, Saad, Sabour, Saddik, Safiri, Sahebkar, H. Salem, M. Salem, Salimzadeh, Salomon, Samy, Sanabria, Santric Milicevic, Sartorius, Sarveazad, Sathian, Satpathy, Savic, Sawhney, Sayyah, Schneider, Schöttker, Sekerija, Sepanlou, Sepehrimanesh, Seyedmousavi, Shaahmadi, Shabaninejad, Shahbaz, Shaikh, Shamshirian, Shamsizadeh, H. Sharafi, Z. Sharafi, Sharif, A. Sharifi, H. Sharifi, Sharma, Sheikh, Shirkoohi, Shukla, Si, Siabani, Silva, Silveira, A. Singh, J. Singh, Sisay, Sitas, Sobngwi, Soofi, Soriano, Stathopoulou, Sufiyan, Tabarés-Seisdedos, Tabuchi, Takahashi, Tamtaji, Tarawneh, Taymoori, Tehrani-Banihashemi, M. Temsah, O. Temsah, B. Tesfay, F. Tesfay, Teshale, Tessema, Thapa, Tlaye, Topor-Madry, Tovani-Palone, Traini, B. Tran, K. Tran, Tsadik, Ullah,
Uthman, Vacante, Vaezi, Varona Pérez, Vidale, Violante, Vlassov, Vollset, Vos, Vosoughi, Vu, Vujcic, Wachamo, Wagnew, Waheed, Weldegebreal, Weldesamuel, Wijeratne, Wondafrash, Wonde, Wondmieneh, Workie, Yadav, Yadegar, Yadollahpour, Yaseri, Yazdi-Feyzabadi, Yeshaneh, Yimam, Yimer, Yisma, Yonemoto, Younis, Yousefifard, Yu, Zabeh, Zadnik, Zahirian Moghadam, Zaidi, Zamani, Zandian, Zangeneh, Zaki, Zendehdel, Zenebe, Zewale, Ziapour, Zodpey, Murray.

Statistical analysis: Fitzmaurice, Abate, Abbastabar, Abdulle, Abraha, Adedeji, Alemnew, Alene, Aljunid, Alsowaidi, Andrei, Ansari, Aremu, Atalay, Ausloos, Awoke, Bagherzadeh, Battista, Bekele, Belay, Bin Sayeed, Bogale, Chaturvedi, Chehrazi, Constantin, Damiani, Demis, Demissie, Deribe, Endalew, Endries, Farzadfar, Feyissa, Fisher, Gedefaw, Gelaw, Getachew, Ghajar, Girmay, Arvin Haj-Mirzaian, Arya Haj-Mirzaian, Hassen, S. Hay, Henry, Herteliu, Hidru, Hoang, Hosseinzadeh, Hussen, Ileanu, Irvani, Iseh, James, Karch, Kasaeian, Khater, Khayamzadeh, Khazaei, Khubchandani, A. Kisa, S. Kisa, Lake, Leigh, Limenih, Lopukhov, Manamo, Mansournia, Mantovani, Maswabi, McAlinden, Meier, H. Meles, Melku, Mohammad Darwesh, A. Mohammed, S. Mohammed, Mokdad,

Moradi-Lakeh, Muche, Muhammed, Nagarajan, Neupane, Ngunjiri, Nguyen, Nnaji, Otstavnov, Pana, Varona Pérez, Pirestani, Prakash, Qorbani, M. Rabiee, N. Rabiee, Rafiei, Rana, Raoofi, Reiner, Sabbagh, Safiri, M. Salem, Samy, Sathian, Satpathy, Shaikh, Silva, Tarawneh, Tassew, B. Tesfay, Teshale, Tlaye, B. Tran, Uthman, Vacante, Vos, Vu, Wachamo, Weldegebreal, Weldesamuel, Wonde, Workie, Yadegar, Yu, Zabeh, Zaidi, Zenebe, Zewale, Ziapour.

Obtained funding: Bagherzadeh, A. Briko, N. Briko, Iseh, Lauriola, Mokdad, Nahvijou, N. Rabiee, Sabbagh, Satpathy.

Administrative, technical, or material support: Abd-Allah, Adedeji, Afarideh, Agius, Ahmadi, Akinyemiju, AlAbdulKader, Alam, Alipour, Aljunid, Allah Bakeshei, S. Amini, Amoako, Anbari, Ansariadi, Appiah, Arab-Zozani, Areri, Assadi, Atique, Awasthi, Bacha, Badawi, Berhane, C. Castro, F. Castro, Catalá-López, Chattu, Chaturvedi, Chiang, Dahlwai, Daryani, das Neves, Demissie,

Denova-Gutiérrez, Desalegn, Doku, Dubey, El Razek, El Sayed Zaki, Emamian, Faramarzi, Farzadfar, Fentahun, Gankpe, P. Gupta, R. Gupta, R. Hay, Househ, Irvani, Jafari Balalami, Jenabi, Joo Kamangar, Kasahun, Khalilarjmandi, Khosravi, Y. Kim, Krohn, Kumar, Liao, Lopez, Malekzadeh, N. Manafi, Mansourian, Merat, Moazen,

Mohammadbeigi, M. Mohammadi, Mohammadian, Mohammadian-Hafshejani, Mohammadpourhodki S. Mohammed, Moradpour, Morgado-da-Costa, Morisaki, Nagarajan, Najafi, Nazari, Nggada, Ningrum, Nourollahpour Shiadeh, Obsa, Oluwasanu, Omonisi, P A, Patil, Pennini, Pereira, M. Rabiee, Radfar, Rafiei, Rahim, Raoofi, Rath, A. Ribeiro, Roro, Rostami, Saad, Sabbagh, Samy, Satpathy, Sayyah, Seyedmousavi, Shamsizadeh, Si Siabani, Silveira, Soriano, B. Tesfay, Wabinga, Wijeratne, Wondmieneh, Yadav, Yadegar, Yadollahpour, Younis, Zahirian Moghadam, Zandian, Zangeneh.

Study supervision: Abualhasan, Alam, E. Amini, S. Amini, Anbari, Ataeinia, Atalay, Atique, Masoud Behzadifar, Meysam Behzadifar, Berfield, Daryani, Demoz, Dinberu, Drake, Dubljanin, El Sayed, El Sayed Zaki, Fadhil, Fukumoto, Gayesa,
Gebremeskel, Ghafourifard, Gizaw, Guimaraes, Hadkhale, Irvani, Iseh, James, Jee, Karch, Khader, Khater, Kianipour, Koyanagi, Krohn, N. Manafi, Merat, B. Miazgowski, T. Miazgowski, Mills, Moazen, M. Mohammadi, A. Mohammed, Mokdad, Nabhan, Nagel, Nazari, Nixon, Obsa, Ota, Pakpour, Pana, Park, Piccinelli, Postma, Pourshams, D. Rawaf, Rezaei, H. Salem, Salomon, Sarveazad, Satpathy, Sepehrimanesh, Silveira, Soriano, Tabuchi, B. Tesfay, Ullah, Vidale, Vos, Vujcic, Zabeh, Zandian, Murray.

Other-Provided expert opinion on regional trends: Diaz. Other-Future and further work: Nagarajan. Other-Advice on analysis and reporting strategy: Sitas.

Other-Provided suggestions for future directions: Owolabi, Pakhale.

Other-Advice on policy implications: Majeed.

Conflict of Interest Disclosures: Dr Goulart reports receiving grants from the National Council for Scientific and Technological Development. Dr Haro reports being a contractor for Eli Lilly and Company and receiving personal fees from Lundbeck. $\mathrm{Mr}$ Hidru reports receiving grants, personal fees, nonfinancial support, and other support from Adigrat University. Dr Iseh reports receiving nonfinancial support from the Usmanu Danfodiyo University Teaching Hospital and the Institute of Human Virology, Nigeria. Dr Jakovljevic reports receiving grants from the Ministry of Education, Science and Technological Development of the Republic of Serbia. Dr James reports receiving grants from Sanofi Pasteur. Dr Lazarus reports receiving grants and personal fees from AbbVie, Gilead Sciences, and MSD. Dr Meretoja reports receiving grants from the Cancer Foundation Finland. Dr Moradi-Lakeh reports being a consultant for a project funded by Novartis. Dr Postma reports receiving grants from Quintiles and Bayer; personal fees from AbbVie, Astellas, and Pharmerit; grants and personal fees from Bristol-Myers Squibb, AstraZeneca, Sanofi, Novartis, Janssen, GlaxoSmithKline, Pfizer, MSD, and Asc Academics, as well as serving as an advisor for the organization; and earnings from stock in Ingress Health and PAB BV. Dr Savic reports being an employee of the GlaxoSmithKline group of companies and holds restricted shares in the GlaxoSmithKline group of companies. Dr J. Singh reports receiving personal fees from serving as a consultant for Crealta/Horizon, Medisys, Fidia, UBM LLC, Medscape, WebMD, the National Institutes of Health, and the American College of Rheumatology; earnings from stocks in the Amarin Corporation and Viking Therapeutics; and nonfinancial support from serving as a member of OMERACT, the US Department of Veterans Affairs Rheumatology Field Advisory Committee, and committees of the American College of Rheumatology. No other disclosures are reported.

Funding/Support: The Institute for Health Metrics and Evaluation received funding from the Bill \& Melinda Gates Foundation.

Role of the Funder/Sponsor: The funders had no role in the design and conduct of the study; collection, management, analysis, and interpretation of the data; preparation, review, or approval of the manuscript; and decision to submit the manuscript for publication. 
Additional Contributions: We thank cancer registries worldwide for their efforts in generating data on cancer incidence.

\section{REFERENCES}

1. United Nations General Assembly. Political declaration of the third high-level meeting of the General Assembly on the prevention and control of non-communicable diseases. http://www.un.org/ en/ga/search/view_doc.asp?symbol=A/RES/73/2. Published October 17, 2018. Accessed February 6 2019.

2. United Nations. 2011 High level meeting on prevention and control of non-communicable diseases. http://www.un.org/en/ga/ ncdmeeting2011/.Published September 16, 2011. Accessed September 3, 2019.

3. World Health Organization. Global Action Plan for the Prevention and Control of NCDs 2013-2020. http://www.who.int/nmh/events/ncd_action_plan/ en/. Published 2013. Accessed September 3, 2019.

4. United Nations. Sustainable development goals: knowledge platform. https://

sustainabledevelopment.un.org/. Published January 2016. Accessed September 1, 2016.

5. Fitzmaurice C, Akinyemiju TF, Al Lami FH, et al Global Burden of Disease Cancer Collaboration. Global, regional, and national cancer incidence, mortality, years of life lost, years lived with disability, and disability-adjusted life-years for 29 cancer groups, 1990 to 2016: a systematic analysis for the Global Burden of Disease Study. JAMA Oncol. 2018;4(11):1553-1568. doi:10.1001/jamaoncol.2018. 2706

6. Dicker D, Nguyen G, Abate D, et al; GBD 2017 Mortality Collaborators. Global, regional, and national age-sex-specific mortality and life expectancy, 1950-2017: a systematic analysis for the Global Burden of Disease Study 2017. Lancet. 2018;392(10159):1684-1735. doi:10.1016/S0140-6736 (18)31891-9
7. James SL, Abate D, Abate KH, et al; GBD 2017 Disease and Injury Incidence and Prevalence Collaborators. Global, regional, and national incidence, prevalence, and years lived with disability for 354 diseases and injuries for 195 countries and territories, 1990-2017: a systematic analysis for the Global Burden of Disease Study 2017. Lancet. 2018;392(10159):1789-1858. doi:10. 1016/S0140-6736(18)32279-7

8. $\mathrm{Kyu} \mathrm{HH}$, Abate D, Abate KH, et al; GBD 2017 DALYs and HALE Collaborators. Global, regional, and national disability-adjusted life-years (DALYs) for 359 diseases and injuries and healthy life expectancy (HALE) for 195 countries and territories, 1990-2017: a systematic analysis for the Global Burden of Disease Study 2017. Lancet. 2018;392(10159):1859-1922. doi:10.1016/S0140-6736 (18)32335-3

9. Roth GA, Abate D, Abate KH, et al; GBD 2017 Causes of Death Collaborators. Global, regional, and national age-sex-specific mortality for 282 causes of death in 195 countries and territories, 1980-2017: a systematic analysis for the Global Burden of Disease Study 2017. Lancet. 2018;392(10159):17361788. doi:10.1016/S0140-6736(18)32203-7

10. Foreman KJ, Lozano R, Lopez AD, Murray CJ. Modeling causes of death: an integrated approach using CODEm. Popul Health Metr. 2012;10:1. doi:10 1186/1478-7954-10-1

11. Jensen OM, Estève J, Møller $\mathrm{H}$, Renard $\mathrm{H}$. Cancer in the European Community and its member states. Eur J Cancer. 1990;26(11-12):1167-1256. doi: 10.1016/0277-5379(90)90278-2

12. Ferlay J, Soerjomataram I, Dikshit R, et al. Cancer incidence and mortality worldwide: sources, methods and major patterns in GLOBOCAN 2012. Int J Cancer. 2015;136(5):E359-E386. doi:10.1002/ ijc.29210

13. Fitzmaurice C, Allen C, Baber RM, et al; Global Burden of Disease Cancer Collaboration. Global, regional, and national cancer incidence, mortality, years of life lost, years lived with disability, and disability-adjusted life-years for 32 cancer groups, 1990 to 2015: a systematic analysis for the Global Burden of Disease Study. JAMA Oncol. 2017;3(4) 524-548. doi:10.1001/jamaoncol.2016.5688

14. Fitzmaurice $C$, Dicker D, Pain $A$, et al; Globa Burden of Disease Cancer Collaboration. The global burden of cancer 2013. JAMA Oncol. 2015;1(4): 505-527. doi:10.1001/jamaoncol.2015.0735

15. Bray F, Ferlay J, Soerjomataram I, Siegel RL, Torre LA, Jemal A. Global cancer statistics 2018 GLOBOCAN estimates of incidence and mortality worldwide for 36 cancers in 185 countries. CA Cancer J Clin. 2018;68(6):394-424. doi:10.3322/ caac. 21492

16. Truven Health Analytics. United States MarketScan Commercial Claims and Encounters Database. Ann Arbor, MI. https://truvenhealth.com/ your-healthcare-focus/government/analyticresearch/marketscan. Accessed September 10, 2019.

17. Jan S, Laba T-L, Essue BM, et al. Action to address the household economic burden of non-communicable diseases. Lancet. 2018;391 (10134):2047-2058. doi:10.1016/S0140-6736(18) 30323-4

18. Niessen LW, Mohan D, Akuoku JK, et al. Tackling socioeconomic inequalities and non-communicable diseases in low-income and middle-income countries under the Sustainable Development agenda. Lancet. 2018;391(10134): 2036-2046. doi:10.1016/S0140-6736(18)30482-3

19. World Health Organization. WHO director-general calls for all countries to take action to help end the suffering caused by cervical cancer. https://www.who.int/reproductivehealth/call-toaction-elimination-cervical-cancer/en/. Published May 19, 2018. Accessed September 3, 2019.

20. Siegel RL, Miller KD, Jemal A. Cancer statistics, 2019. CA Cancer J Clin. 2019;69(1):7-34. doi:10. 3322/caac. 21551 